\title{
REVISIÓN SISTEMÁTICA SOBRE LA EVALUACIÓN DE PROPUESTAS DE GAMIFICACIÓN EN SIETE DISCIPLINAS EDUCATIVAS
}

\section{Sistematic Review about Evaluation of Gamification in Seven Educational Disciplines}

Joel Manuel PRIETO ANDREU

Universidad Internacional de la Rioja. España.

joelmanuel.prieto@unir.net

https://orcid.org/0000-0002-2981-0782

Fecha de recepción: 10/07/2021

Fecha de aceptación: 05/09/2021

Fecha de publicación en línea: 01/01/2022

Cómo citar este artículo: Prieto Andreu, J. M. (2022). Revisión sistemática sobre la evaluación de propuestas de gamificación en siete disciplinas educativas. Teoría de la Educación. Revista Interuniversitaria, 34(1), 189-214. https://doi.org/10.14201/teri.27153

RESUMEN

La evaluación de los procesos de gamificación en educación, pueden plantear una alternativa a las estrategias de evaluación tradicionales del aula. Esta revisión sistemática analiza cómo se han evaluado las diferentes propuestas de gamificación en diferentes áreas de conocimiento como ciencias de la salud, ciencias exactas, ciencias sociales y humanidades. Se identificaron ochenta y cinco estudios significativos a través de una búsqueda en ISI Web Of Science y SCOPUS. En esta revisión sistemática se siguieron los criterios establecidos en la declaración PRISMA 2020. La gamificación ha sido evaluada en cuatro áreas de conocimiento distribuidas en siete disciplinas: Educación Física, Fisioterapia y Nutrición, Matemáticas, Física y Química, Informática, Ciencias Sociales, Ciencias Naturales y Lingüística, siendo diferentes los procesos e instrumentos de evaluación que se han empleado en su implantación. Se 
JOEL MANUEL PRIETO ANDREU

REVISIÓN SISTEMÁTICA SOBRE LA EVALUACIÓN DE PROPUESTAS DE GAMIFICACIÓN EN SIETE DISCIPLINAS EDUCATIVAS

señalan los resultados sobre la producción científica, analizando las características de las propuestas gamificadas, el diseño, método y corte de investigación empleado, los resultados motivacionales y de aprendizaje, así como la calidad de la producción científica en cada disciplina educativa mediante la Escala de Evaluación de Artículos Científicos (EACSH) de López et al. (2019). La escala está compuesta por diecinueve criterios de evaluación concretados en ocho dimensiones: resumen, introducción, metodología, resultados, discusión, referencias, apéndices, estilo y formato. Se concluye que las variables psicológicas que más se evaluaron en las propuestas gamificadas fueron el aprendizaje y la motivación, siendo todos los resultados positivos. Por otro lado, se establecieron correlaciones significativas entre los instrumentos de evaluación y la mejora de alguna variable psicológica: grupos de reflexión y participación, cuestionarios y motivación, entrevistas y atención, test de evaluación y rendimiento, y encuestas para mejorar la satisfacción. Se analizan y discuten los resultados en cada una de las siete disciplinas estudiadas.

Palabras clave: evaluación; gamificación; educación; revisión sistemática; ciencias; sociales; salud; lingüística.

\section{ABSTRACT}

The evaluation of gamification processes in education can pose an alternative to traditional classroom evaluation strategies. This systematic review analyzes how the different gamification proposals have been evaluated in different areas of knowledge such as health sciences, exact sciences, social sciences and humanities. Eighty-five significant studies were identified through a search of ISI Web Of Science and SCOPUS. In this systematic review, the criteria established in the PRISMA 2020 statement were followed. Gamification has been placed in four areas of knowledge distributed in seven disciplines: Physical Education, Physiotherapy and Nutrition, Mathematics, Physics and Chemistry, Computer Science, Social Sciences, Natural Sciences and Linguistics, the evaluation processes and instruments that have been used in its implementation are different. The results on the scientific production are indicated, analyzing the characteristics of the gamified proposals, the design, method and cut of the research used, the motivational and learning results, as well as the quality of the scientific production in each educational discipline through the Scale of Evaluation of Scientific Articles (EACSH) by López et al. (2019). The scale is made up of nineteen evaluation criteria specified in eight dimensions: summary, introduction, methodology, results, discussion, references, appendices, style and format. It is concluded that the psychological variables that were most evaluated in the gamified proposals were learning and motivation, with all positive results. On the other hand, significant correlations were established between the evaluation instruments and the improvement of some psychological variable: reflection and participation groups, questionnaires and motivation, interviews and attention, evaluation and performance tests, and surveys to improve satisfaction. The results are analyzed and discussed in each of the seven disciplines studied.

Key words: evaluation; gamification; education; systematic review; science; social; health; linguistics. 

EN SIETE DISCIPLINAS EDUCATIVAS

\section{INTRODUCCIÓN}

Los estudios que han evaluado los procesos de gamificación parten de la asunción de que los juegos con soporte digital promueven la motivación de los usuarios, ya que pueden producir el aprendizaje, incluso en una perspectiva tangencial (Alshammari, 2020; Hernando et al., 2015; Ling, 2018; Mora-González et al, 2020; Pérez-López et al., 2017; Quintero et al., 2018; Rodríguez-Oroz et al., 2019; Sevilla y Haba, 2017). Sin embargo, realizar una actividad pedagógica gamificada no implica crear un juego con un sesgo pedagógico, solo es necesario comprender el uso de las mecánicas y las dinámicas presentes en los juegos digitales, impregnando las prácticas pedagógicas de dichos elementos (Prieto, 2020).

En esta línea, Leitão et al. (2021) indican que la motivación y el compromiso son algunos de los conceptos más comúnmente mencionados y evaluados en gamificación, lo que demuestra que es cada vez más necesario unir (o redescubrir) la alegría y el aprendizaje, sin llegar a eliminar el tema de la diversión en la educación, ni mucho menos de trivializar tal concepto. Junto con la gamificación, los estudios combinan dicha metodología con otras alternativas como los Juegos Serios (Serious Games) o el Aprendizaje Basado en Juegos (Game Based Learning, GBL).

Siguiendo las conclusiones de Colomo-Magaña et al. (2020) la evaluación gamificada es mejor valorada por el alumnado y mejor percibida por los docentes, respecto a la motivación del alumnado, su rendimiento, interés, capacidad de atención, por la continuidad del aprendizaje fuera del aula (ubicuidad) y por su propia satisfacción al implementar la prueba. Pese a que son múltiples los instrumentos (portafolios, cuestionarios, encuestas, entre otros) y metodologías (aprendizaje basado en proyectos, flipped classroom, gamificación, juegos serios, GBL) que se pueden aplicar para evaluar los procesos de aprendizaje del alumnado, el examen sigue siendo la prueba más implementada (Miralles y Monteagudo, 2019).

Existe una gran cantidad de recursos online que gamifican el proceso de evaluación tipo test (Quizizz, Kahoot!, Plickers, Socrative, Goolge Forms, QuizWorks, Qstream, Riddle, Testmoz, aPreguntar), todos de acceso libre y que se pueden emplear en el aula como juegos serios que presentan un fin educativo (Ucar y Kumtepe, 2017).

Existen diversas revisiones de la bibliografía sobre gamificación en el contexto educativo, se estudia la comprensión de los factores que pueden influir en el éxito de un proceso gamificado (Nair y Mathew, 2021), la investigación empírica del uso de la gamificación en algún área educativa (Holguín et al., 2020; Kalogiannakis et al., 2021), o revisiones bibliométricas sobre gamificación en el contexto educativo (Swacha, 2021), aunque no se ha elaborado ninguna revisión que analice la literatura sobre la evaluación llevada a cabo en propuestas didácticas gamificadas en diferentes áreas de conocimiento o disciplinas educativas.

En la Figura 1 se analizan las tres revisiones mencionadas en una infografía, destacando diez preguntas de investigación o hipótesis formuladas en cada una de ellas junto con sus resoluciones. 


\section{FIGURA 1}

DIEZ PREGUNTAS DE INVESTIGACIÓN FORMULADAS EN TRES REVISIONES ACTUALES SOBRE GAMIFICACIÓN EDUCATIVA

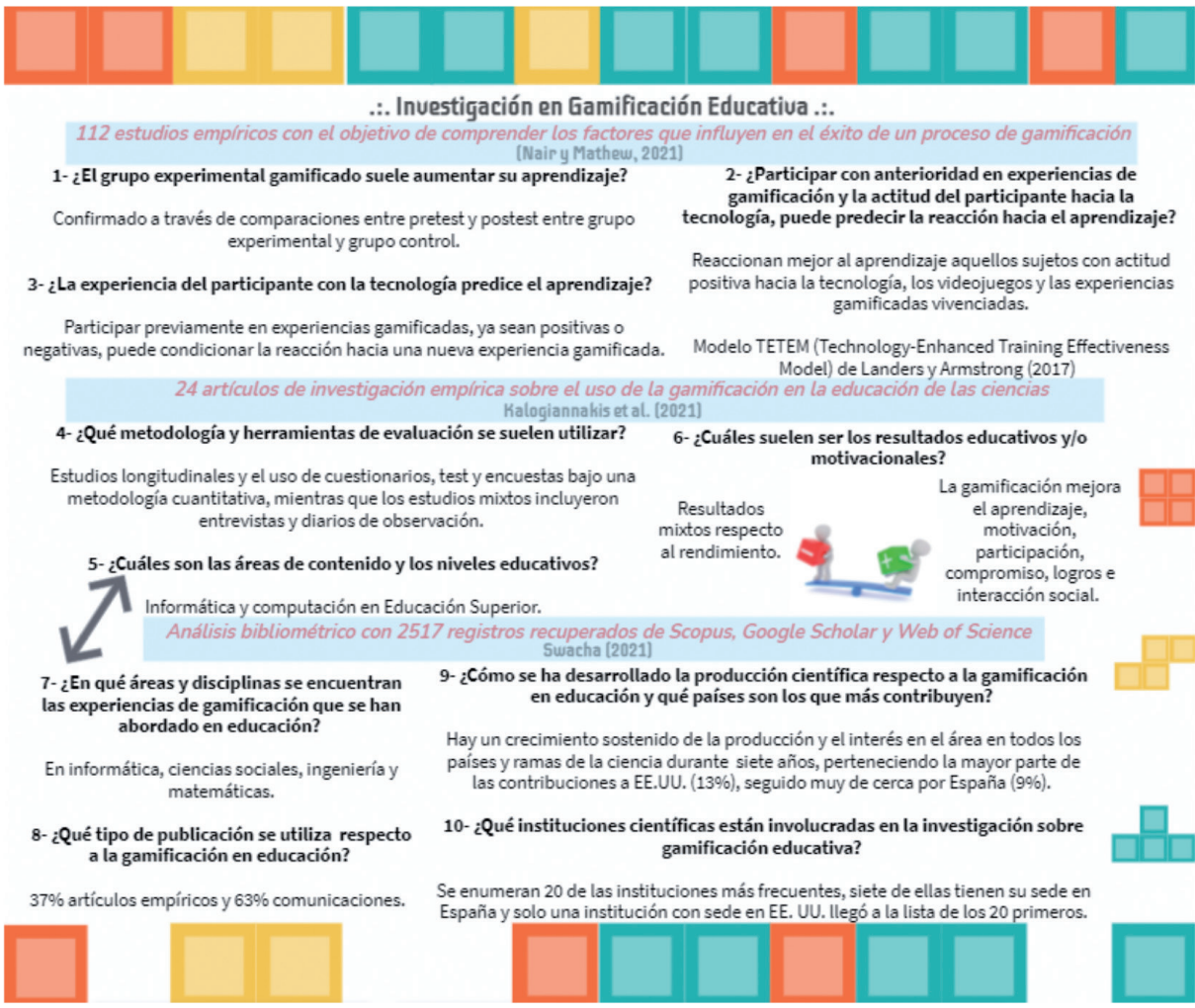

Fuente: Elaboración propia

Para lograr el objetivo propuesto "conocer la evaluación y los resultados motivacionales y de aprendizaje de propuestas gamificadas en diferentes áreas de conocimiento", se analiza la producción científica en siete disciplinas educativas.

\section{MATERIAL Y MÉTODOS}

En la presente revisión sistemática se siguieron los criterios establecidos en la declaración PRISMA (Preferred Reporting Items for Systematic reviews and MetaAnalyses) (Moher et al., 2009), consistente en una lista de comprobación estructurada de 27 ítems. La búsqueda de la información ha estado orientada hacia los estudios relacionados con la evaluación de propuestas didácticas o experiencias de 
gamificación en cuatro áreas de conocimiento: (1) Ciencias de la Salud, (2) Ciencias Exactas, (3) Ciencias Naturales y Sociales, y (4) Humanidades. La localización de artículos se realizó en agosto del 2021 en ISI Web Of Science y en Scopus. La tabla 1 muestra el detalle de la cadena de búsqueda con cada una de las áreas de conocimiento y su composición, según las disciplinas que fueron definidas en la revisión. Se filtró la búsqueda en título, resumen y palabras clave tanto en WOS (TOPIC) como en SCOPUS (TITLE-ABS-KEY).

TABLA 1

CADENAS DE BÚSQUEDA EMPLEADAS EN WOS Y SCOPUS Y PROPORCIÓN DE TRABAJOS RESULTANTES POR AÑO

\begin{tabular}{|c|c|c|c|c|c|}
\hline Año & $\begin{array}{c}\text { Base de } \\
\text { datos }\end{array}$ & $\begin{array}{c}\text { Ciencias } \\
\text { de la Salud } \\
\text { (Gamification } \\
\text { AND Education } \\
\text { OR learning } \\
\text { AND evaluation } \\
\text { AND physiology } \\
\text { OR nutrition } \\
\text { OR physical } \\
\text { education) }\end{array}$ & $\begin{array}{c}\text { Ciencias } \\
\text { Exactas } \\
\text { (Gamification } \\
\text { AND Education } \\
\text { OR learning } \\
\text { AND evaluation } \\
\text { AND Maths } \\
\text { OR physics OR } \\
\text { chemistry OR } \\
\text { computing) }\end{array}$ & $\begin{array}{c}\text { Ciencias } \\
\text { Naturales y } \\
\text { Sociales } \\
\text { (Gamification } \\
\text { AND Education } \\
\text { OR learning } \\
\text { AND evaluation } \\
\text { AND geology } \\
\text { OR biology OR } \\
\text { geography OR } \\
\text { history) } \\
\end{array}$ & $\begin{array}{l}\text { Humanidades } \\
\text { (Gamification } \\
\text { AND Education } \\
\text { OR learning } \\
\text { AND evaluation } \\
\text { AND language } \\
\text { OR literature) }\end{array}$ \\
\hline \multirow{2}{*}{2015} & WOS & 226 & 161 & 234 & 468 \\
\hline & SCOPUS & 2 & 6 & 1 & 5 \\
\hline \multirow[t]{2}{*}{2016} & wOS & 280 & 234 & 360 & 595 \\
\hline & SCOPUS & 1 & 21 & 2 & 3 \\
\hline \multirow[t]{2}{*}{2017} & WOS & 334 & 297 & 472 & 708 \\
\hline & SCOPUS & 5 & 29 & 3 & 14 \\
\hline \multirow[t]{2}{*}{2018} & WOS & 408 & 273 & 525 & 794 \\
\hline & SCOPUS & 2 & 38 & 2 & 8 \\
\hline \multirow[t]{2}{*}{2019} & WOS & 543 & 391 & 625 & 1056 \\
\hline & SCOPUS & 8 & 51 & 8 & 16 \\
\hline \multirow[t]{2}{*}{2020} & WOS & 478 & 429 & 583 & 997 \\
\hline & SCOPUS & 11 & 45 & 8 & 20 \\
\hline \multirow{2}{*}{2021} & WOS & 277 & 224 & 356 & 576 \\
\hline & SCOPUS & 4 & 27 & 4 & 6 \\
\hline
\end{tabular}

Fuente: Elaboración propia, a partir de los datos extraídos en WoS y SCOPUS

En total, se identificaron 13254 registros en ambas bases de datos desde el 2015 hasta agosto del 2021 (12 904 en WOS y 350 en SCOPUS). De este total, se hizo una selección aleatoria de 1128 registros, distribuidos de forma estratificada, con el $95 \%$ de confianza y +/- 4,3 de error muestral (Tabla 2). 
JOEL MANUEL PRIETO ANDREU

REVISIÓN SISTEMÁTICA SOBRE LA EVALUACIÓN DE PROPUESTAS DE GAMIFICACIÓN EN SIETE DISCIPLINAS EDUCATIVAS

TABLA 2

PROPORCIÓN DE TRABAJOS RESULTANTES DE LA BÚSQUEDA DE ARTÍ́CUlOS DE ACCESO LIBRE EN WOS Y SCOPUS

\begin{tabular}{ccccc}
\hline Año & Artículos WOS & $\begin{array}{c}\text { Artículos } \\
\text { SCOPUS }\end{array}$ & $\begin{array}{c}\text { Muestra total } \\
\text { del estrato }\end{array}$ & $\begin{array}{c}\text { Proporción del } \\
\text { estrato }\end{array}$ \\
\hline 2021 & 1.433 & 41 & 94 & $8,3 \%$ \\
2020 & 2.487 & 84 & 245 & $21,7 \%$ \\
2019 & 2.615 & 83 & 264 & $23,4 \%$ \\
2018 & 2.000 & 50 & 206 & $18,2 \%$ \\
2017 & 1.811 & 51 & 169 & $15 \%$ \\
2016 & 1.469 & 27 & 86 & $7,7 \%$ \\
2015 & 1.089 & 14 & 64 & $5,7 \%$ \\
\hline
\end{tabular}

Fuente: Elaboración propia, a partir de los datos extraídos en WoS y SCOPUS

Esta selección se hizo a partir de una tabla de números aleatoria creada ad-hoc, la cual sirvió para seleccionar cada caso de estudio por año y por base de datos, ordenándose por año y asignándose a estos un número único de identificación que permitió la selección de cada unidad muestral. De los estudios que se consideraron resultaron 569 artículos completos para revisión en la fase de clasificación, descartándose por el título, abstract y texto íntegro, los estudios que no se relacionaban con el área de conocimiento o que estudiaban otras variables. Posteriormente, se excluyeron artículos que relacionaban diferentes variables ajenas a la temática de la revisión, por ser estudios epidemiológicos o por no cumplir los criterios de inclusión. De esta manera quedaron 157 estudios en la fase de elegibilidad. Para la selección de los estudios se han tenido en cuenta los siguientes criterios de exclusión: trabajos procedentes de fuentes académicas no confiables; referencias anteriores al 2015; trabajos pertenecientes a repositorios institucionales; publicaciones como artículos de revistas de divulgación, revisiones, tesis o presentaciones en eventos académicos; artículos que estudien la gamificación en ámbitos empresariales, marketing, organizaciones, etc. Por otra parte, para la selección de la muestra de estudio se han tenido en cuenta los siguientes criterios de inclusión: trabajos publicados del año 2015 en adelante, escritos en español y en inglés; artículos revisados por pares y comunicaciones de congresos publicadas en libro de actas; artículos pertenecientes a la categoría: Education and Educational Research en WOS y en cualquier categoría en SCOPUS; trabajos con acceso libre a texto completo en WOS, y otros obtenidos previa solicitud a los autores; y estudios de grupo único y doble comparativo, con diseño de encuesta que valorasen la implantación de la experiencia de gamificación. Posteriormente, en la fase de inclusión se eliminaron 72 artículos, incluyéndose los artículos por texto integral. Del subtotal de los registros encontrados en WOS y SCOPUS se analizaron finalmente 85 estudios (Figura 2), seleccionados intencionalmente, de acuerdo con el referente PICR (Participantes / Intervención / Comparación 
/ Resultados), siendo la población de estudio alumnado de cualquier etapa educativa (Educación Primaria, Secundaria y Superior). En cuanto al criterio de intervención, se seleccionaron estudios experimentales y observacionales, descartándose estudios epidemiológicos, monográficos y patentes. Respecto al criterio de comparación, se exploraron estudios de grupo único, incluyéndose aquellos de dos grupos con pretest y postest. En cuanto al criterio de resultados, se tuvieron en cuenta aquellos estudios que mostraran la evaluación de experiencias de gamificación en cualquiera de las siete disciplinas estudiadas en la revisión sistemática. En la figura 2 se puede observar el flujograma del proceso de revisión siguiendo la declaración PRISMA.

FIGURA 2

FLUJOGRAMA DEL PROCESO DE REVISIÓN SIGUIENDO LA DECLARACIÓN PRISMA

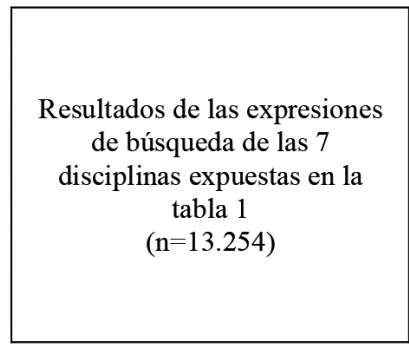

Registros distribuidos de forma estratificada, con el $95 \%$ de confianza $y+/-4,3$ de error en las 2 bases de datos desde el 2015. $(\mathrm{n}=1.128)$

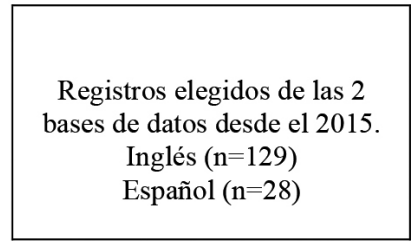

Registros incluidos en la revision sistemática: Inglés $(\mathrm{n}=68)$

Español $(n=17)$

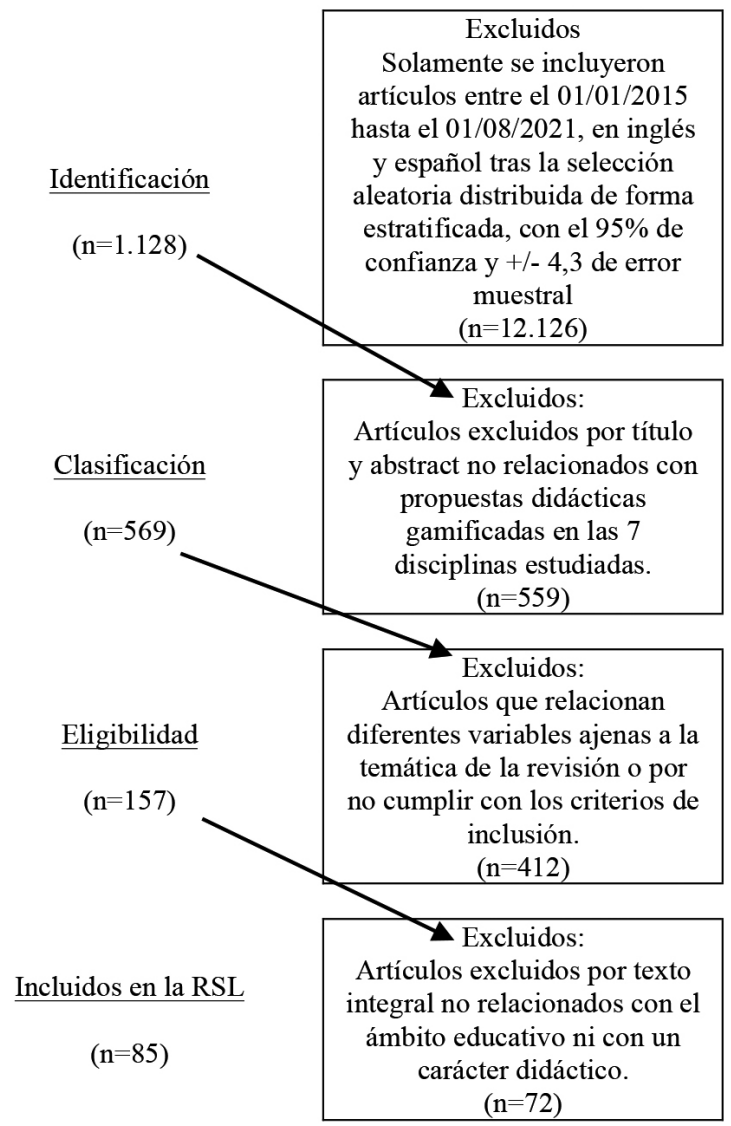

Fuente: Elaboración propia 
JOEL MANUEL PRIETO ANDREU

REVISIÓN SISTEMÁTICA SOBRE LA EVALUACIÓN DE PROPUESTAS DE GAMIFICACIÓN EN SIETE DISCIPLINAS EDUCATIVAS

Para analizar la producción científica se utilizó la Escala para Evaluar Artículos Científicos en Ciencias Sociales y Humanas (EACSH) de López et al. (2019) publicada en una revista situada en el Q1 del SJR y Q2 del JCR, conformada por 19 ítems con una confiabilidad de 0,937 , apropiada para guiar la escritura y evaluación de artículos científicos de tipo cuantitativo con alcance exploratorio y descriptivo, aplicable a las ciencias sociales y humanas, y, específicamente en el ámbito de educación, siendo útil para evaluar los artículos incluidos en la revisión sistemática ya que todos pertenecen al área de "Education and Educational Research" y las revistas donde se han publicado los artículos están indexadas, al menos, en la categoría "Education". De acuerdo con la calidad de la información extraída en los estudios y su contribución al estudio de la gamificación, se plantean 19 criterios de evaluación concretados en 8 dimensiones (resumen: 3 ítems; introducción: 2 ítems; metodología: 4 ítems; resultados: 3 ítems; discusión: 3 ítems; referencias: 1 ítem; apéndices: 1 ítem; estilo y formato: 2 ítems), siendo 19 puntos (20\%) la mínima puntuación que puede obtener la calificación del artículo, y 95 puntos (100\%) la máxima puntuación, siguiendo la siguiente escala de valoración: $1=$ nivel; muy bajo; $2=$ nivel bajo; $3=$ nivel medio; $4=$ nivel medio alto; y $5=$ nivel muy alto. Las pruebas de normalidad de Chi cuadrado y Kolmogorov-Smirnov determinan una distribución no parámetrica en todas las variables ( $\mathrm{p}>$.0.5). Para los cálculos inferenciales se utilizó la prueba de Chi-cuadrado para variables categóricas y Tau-B de Kendal y Rho de Spearman para las correlaciones bivariadas entre variables escalares.

\section{ANÁLISIS Y RESULTAdOS}

Una vez terminadas las fases de planificación, búsqueda y revisión, en primer lugar, se analiza el total de la muestra seleccionada, siendo 85 (80 \% en inglés y $20 \%$ en español) los estudios analizados en la revisión, siendo siete las disciplinas educativas subyacentes de las cuatro áreas de conocimiento en las que se centró la revisión: Educación Física (46,2 \% en inglés y 53,8 \% en español), Fisioterapia y Nutrición (87,5 \% en inglés y 12,5 \% en español), Matemáticas, Física y Química (87,5 \% en inglés y 12,5 \% en español), Informática (89,5 \% en inglés y 10,5\% en español), Ciencias Sociales (88,9 \% en inglés y 11,1 \% en español), Ciencias Naturales $(72,7 \%$ en inglés y $27,3 \%$ en español) y Lingüística (88,2 \% en inglés y 11,8 $\%$ en español). En la tabla 3 se puede observar el total de artículos analizados en cada disciplina educativa.

En segundo lugar, de acuerdo con la calidad de la información extraída en los estudios y su contribución al estudio de la gamificación, en la Figura 3 se relacionan las 7 disciplinas educativas con la calidad de su contribución respecto a las propuestas didácticas de gamificación analizadas. De acuerdo con los análisis de la contribución científica, la calidad no bajo del 53,7 \%, puesto que el cuerpo base del estudio se encuentra publicado en revistas de prestigio, en su mayoría indexadas en algún cuartil del ranking JCR. Se determinaron 3 categorías de calidad con 

EN SIETE DISCIPLINAS EDUCATIVAS

base en los criterios de la Escala EACSH de López et al. (2019), estableciendo los siguientes puntos de corte: Calidad Alta (87,4 \% - $100 \%)$ aquellas contribuciones sin recomendaciones, apéndices o discusión limitada; Calidad Media (68,4 \% - 83,1 \%) además de lo anterior, contribuciones con metodología e introducción regular cuyos resultados se deben complementar con tablas o figuras; y Calidad Baja (53,7 $\%-62,1 \%$ ) aquellas contribuciones con discusión muy limitada, introducción y metodología regular y menor aporte al área de conocimiento o disciplina educativa.

TABLA 3

CUERPO BASE DEL ESTUDIO DISTRIBUIDO EN SIETE DISCIPLINAS EDUCATIVAS

\begin{tabular}{ccc}
\hline Disciplinas educativas & Artículos & $\mathbf{\%}$ \\
\hline Educación Física & 13 & $14,12 \%$ \\
Fisioterapia y Nutrición & 8 & $10,59 \%$ \\
Matemáticas, Física y Química & 8 & $9,41 \%$ \\
Informática & 19 & $22,35 \%$ \\
Ciencias Sociales & 9 & $10,59 \%$ \\
Ciencias Naturales & 11 & $12,94 \%$ \\
Lingüística & 17 & $20 \%$ \\
\hline
\end{tabular}

Fuente: Elaboración propia

FIGURA 3

CALIDAD DE LA PRODUCCIÓN CIENTÍFICA REVISADA POR DISCIPLINA EDUCATIVA

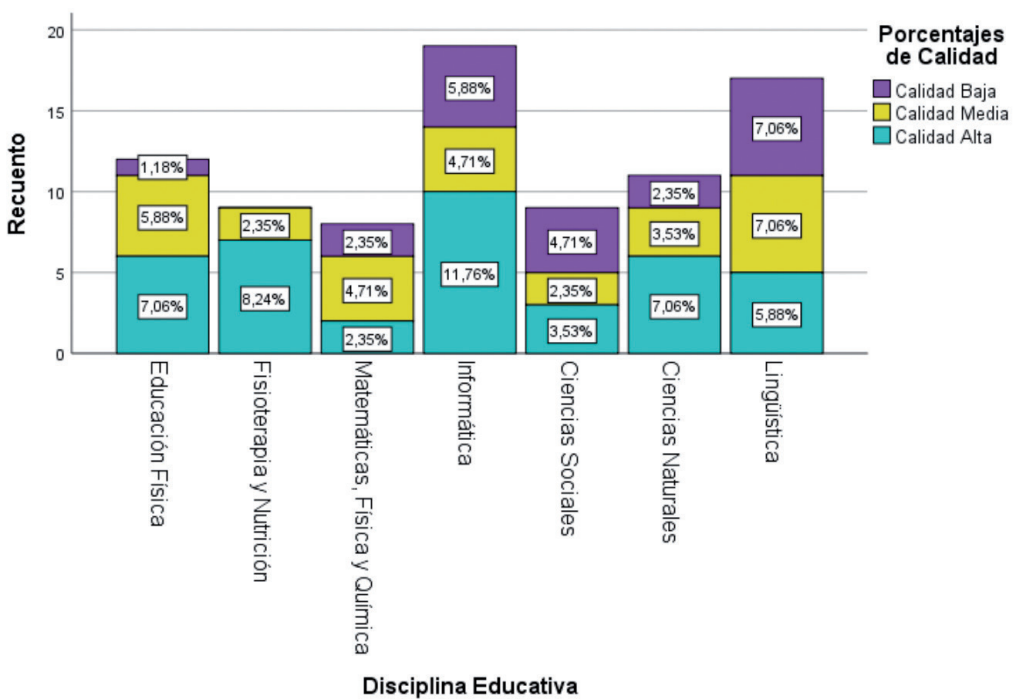

Fuente: Elaboración propia basada en los criterios de la Escala EACSH de López et al. (2019) 
JOEL MANUEL PRIETO ANDREU

REVISIÓN SISTEMÁTICA SOBRE LA EVALUACIÓN DE PROPUESTAS DE GAMIFICACIÓN EN SIETE DISCIPLINAS EDUCATIVAS

En la figura 3 se puede observar que Informática es la disciplina educativa con mayor calidad (11,76\%) en proporción al cuerpo base del estudio, siendo Ciencias Sociales (4,71\%) y Lingüística (7,06 \%) las disciplinas que reportan una calidad más baja. Respecto a la citación, se han contabilizado las citas que ha recibido cada disciplina educativa en proporción a los estudios incluidos en la revisión. En la tabla 4 se detallan los estadísticos descriptivos, siendo Informática la disciplina educativa que más citas ha recibido (29,56 \%), seguida de Educación Física $(18,11$ \%) y Ciencias Sociales (16,61\%). La correlación bivariada entre las citas y los porcentajes de calidad no arrojó resultados significativos al aplicar las pruebas Tau-B de Kendal y Rho de Spearman ( $\mathrm{p}>.05)$.

TABLA 4

ESTADÍSTICOS DESCRIPTIVOS DE LAS CITAS RECIBIDAS POR DISCIPLINA EDUCATIVA

\begin{tabular}{ccccc}
\hline Disciplina & Media & Mediana & $\begin{array}{c}\text { Desviación } \\
\text { estándar }\end{array}$ & Porcentaje \\
\hline Educación Física & 23,25 & 13,50 & 23,43 & $18,11 \%$ \\
Fisioterapia y Nutrición & 11,78 & 8,00 & 18,19 & $9,17 \%$ \\
Matemáticas, Física y Química & 8,75 & 2,00 & 11,03 & $6,82 \%$ \\
Informática & 37,95 & 7,00 & 61,28 & $29,56 \%$ \\
Ciencias Sociales & 21,33 & 8,00 & 42,26 & $16,61 \%$ \\
Ciencias Naturales & 8,82 & 5,00 & 11,71 & $6,90 \%$ \\
Lingüística & 16,47 & 17,00 & 17,55 & $12,83 \%$ \\
\hline
\end{tabular}

Fuente: Elaboración propia

En tercer lugar, se ha analizado el tipo de diseño, corte y método de los estudios analizados. Una vez analizado el cuerpo base del estudio, se resalta que el $60 \%$ de las propuestas son experimentales, un 24,7 \% observacionales y un $15,3 \%$ cuasi-experimentales, priorizando las de corte longitudinal $(67,1 \%)$ frente a las de corte transversal (32,9\%), bajo un método mayormente cuantitativo (54,1\%) frente aquellas propuestas con un corte mixto (28,2 \%) o cualitativo (17,6 \%). En la tabla 5 se puede observar el tipo de diseño, corte y método que tuvieron las propuestas gamificadas en las siete disciplinas educativas. En todas las categorías se obtuvo una significación de $\mathrm{p}<.05$ al aplicar la prueba Chi-cuadrado para variables categóricas. 
JOEL MANUEL PRIETO ANDREU

REVISIÓN SISTEMÁTICA SOBRE LA EVALUACIÓN DE PROPUESTAS DE GAMIFICACIÓN

EN SIETE DISCIPLINAS EDUCATIVAS

TABLA 5

PRODUCCIÓN CIENTÍFICA DISTRIBUIDA EN CADA UNA DE LAS CATEGORÍAS ESTUDIADAS

\begin{tabular}{|c|c|c|c|c|c|c|c|c|}
\hline & & 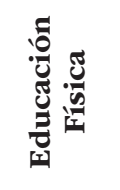 & 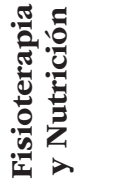 & 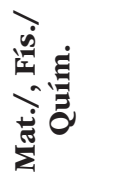 & 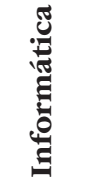 & 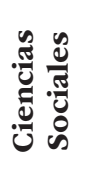 & 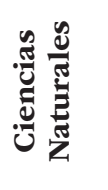 & 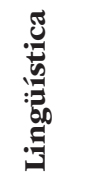 \\
\hline \multirow{3}{*}{ Diseño } & Cuasi-experimental & $58,3 \%$ & $11,1 \%$ & $12,5 \%$ & $10,5 \%$ & $11,1 \%$ & $0,0 \%$ & $5,9 \%$ \\
\hline & Experimental & $33,3 \%$ & $88,9 \%$ & $50,0 \%$ & $84,2 \%$ & $44,4 \%$ & $72,7 \%$ & $41,2 \%$ \\
\hline & Observacional & $8,3 \%$ & $0,0 \%$ & $37,5 \%$ & $5,3 \%$ & $44,4 \%$ & $27,3 \%$ & $52,9 \%$ \\
\hline \multirow{2}{*}{ Corte } & Longitudinal & $100,0 \%$ & $66,7 \%$ & $62,5 \%$ & $57,9 \%$ & $44,4 \%$ & $54,5 \%$ & $76,5 \%$ \\
\hline & Transversal & $0,0 \%$ & $33,3 \%$ & $37,5 \%$ & $42,1 \%$ & $55,6 \%$ & $45,5 \%$ & $23,5 \%$ \\
\hline \multirow{3}{*}{ Método } & Cuantitativo & $33,3 \%$ & $88,9 \%$ & $62,5 \%$ & $68,4 \%$ & $66,7 \%$ & $63,6 \%$ & $17,6 \%$ \\
\hline & Cualitativo & $41,7 \%$ & $11,1 \%$ & $0,0 \%$ & $5,3 \%$ & $11,1 \%$ & $18,2 \%$ & $29,4 \%$ \\
\hline & Mixto & $25,0 \%$ & $0,0 \%$ & $37,5 \%$ & $26,3 \%$ & $22,2 \%$ & $18,2 \%$ & $52,9 \%$ \\
\hline
\end{tabular}

Fuente: Elaboración propia

En cuarto lugar, en la figura 4 se pueden observar los procedimientos e instrumentos de evaluación más empleados por disciplina educativa. Se diferenciaron siete tipos de instrumentos de evaluación bajo dos tipos de procedimientos. Procedimientos Observacionales:1-Registros de juego (25,9\%); 2-Diarios de observaciones o diarios de campo (14,1\%); 3-Grupos de reflexión, grupos de discusión, grupos focales o foros de discusión (5,2 \%). Procedimientos Experimentales: 4-Cuestionarios (29,4 \%); 5-Entrevistas (8,2 \%); 6-Test de evaluación (17,6 \%); y 7-Encuestas (17,6 \%). En todas las categorías se obtuvo una significación de $\mathrm{p}<.05$ al aplicar la prueba Chicuadrado para variables categóricas.

FIGURA 4

INSTRUMENTOS DE EVALUACIÓN EMPLEADOS EN CADA DISCIPLINA EDUCATIVA

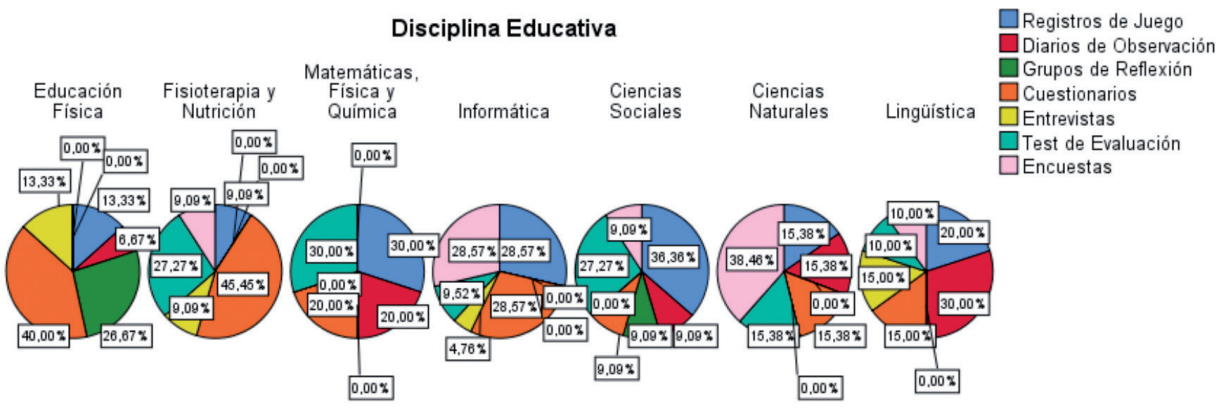

Fuente: Elaboración propia 
JOEL MANUEL PRIETO ANDREU

REVISIÓN SISTEMÁTICA SOBRE LA EVALUACIÓN DE PROPUESTAS DE GAMIFICACIÓN EN SIETE DISCIPLINAS EDUCATIVAS

En quinto lugar, se han analizado los resultados motivacionales y de aprendizaje de los artículos incluidos en la revisión. En la figura 5 se pueden observar, por disciplina educativa, aquellas variables más estudiadas respecto al proceso de gamificación implantado. Se distinguieron ocho resultados motivacionales o de aprendizaje: 1-Aprendizaje, aprendizaje cooperativo o colaborativo, desarrollo de hábitos, competencias, habilidades o adquisición de algún contenido (47,1%); 2-Disfrute o diversión (8,2 \%); 3-Compromiso, adherencia, intención de uso, esfuerzo o actitud positiva (10,6 \%); 4-Atención (1,2\%); 5-Rendimiento o desempeño (9,2\%); 6-Satisfacción (7,1 \%); 7-Interés o utilidad percibida (9,4\%); 8-Participación (8,2 \%); y 9-Motivación (40\%). En todos los resultados motivacionales y de aprendizaje se obtuvo una significación de $\mathrm{p}<.05$ al aplicar la prueba Chi-cuadrado para variables categóricas.

FIGURA 5

RESUlTADOS MOTIVACIONALES O DE APRENDIZAJE EN CADA DISCIPLINA EDUCATIVA

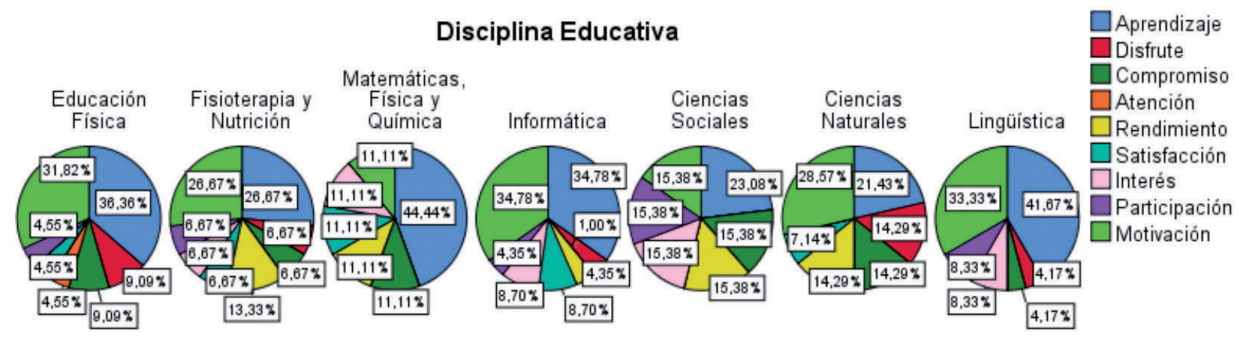

Fuente: Elaboración propia

Se establecieron correlaciones significativas entre los instrumentos de evaluación y los resultados motivacionales y de aprendizaje: grupos de reflexión y participación $(0,289, \mathrm{p}=, 007)$, cuestionarios y motivación $(0,264, \mathrm{p}=, 015)$, entrevistas y atención $(0,364, \mathrm{p}=, 001)$, test de evaluación y rendimiento $(0,591, \mathrm{p}=, 000)$, y encuestas y satisfacción $(0,234, p=, 031)$. Los registros de juego y los diarios de observación no se relacionaron estadísticamente con ningún resultado motivacional o de aprendizaje.

En sexto y último lugar, en la tabla 6 se presenta el listado de los estudios seleccionados en la revisión sistemática en relación con los indicadores estudiados y ordenados por disciplina educativa.

\section{Discusión de LOS RESULTAdoS Y CONCLUSIONES}

La muestra de la revisión sistemática es de 85 producciones científicas, siendo siete las disciplinas educativas subyacentes de las cuatro áreas de conocimiento en las que se centró la revisión. De acuerdo a la calidad de la información extraída 
en los estudios y su contribución al estudio de la gamificación, Informática es la disciplina educativa con mayor calidad en proporción al cuerpo base del estudio. La informática es un tema educativo algo difícil, por lo que se demanda especialmente el apoyo al compromiso y la motivación que ofrece la gamificación al servicio de un profesorado más acostumbrado al entorno digital. Por otro lado, aunque la disciplina de Informática fue la más citada, no hubo relaciones significativas entre citación y calidad de la contribución, por lo cual un artículo poco citado no significa necesariamente que sea de baja calidad. En esta línea, en la revisión bibliométrica de Swacha (2021) se destacó una comunicación de investigación eficaz documentada por el número de citas y por el mapa de co-citación, demostrando que existe una amplia colaboración entre investigadores, siendo los resultados de la gamificación en investigación educativa ampliamente reconocidos.

Respecto al tipo de diseño, corte y método de los estudios analizados, primaron los estudios experimentales de corte longitudinal bajo métodos cuantitativos o mixtos en la mayoría de las disciplinas, destacando en el 15,3\% de los estudios la comparación entre grupo de control y grupo experimental gamificado. Se resalta el uso de un diseño cuasi-experimental en Educación Física (58,3 \%) y observacional $(52,9 \%)$ en Lingüística, destacando el uso de un corte longitudinal en todas las propuestas de Educación Física, siendo Ciencias Sociales la única disciplina que empleó con mayor asiduidad un corte transversal (55,6 \%). Por otro lado, destacan las disciplinas de Lingüística (52,9 \%) y Educación Física (41,7 \%) usando métodos mixtos y cualitativos, respectivamente.

Por otro lado, en esta revisión se diferenciaron procedimientos observacionales y experimentales, resaltando los instrumentos de evaluación más empleados por disciplina educativa. Los instrumentos de evaluación más empleados en la totalidad de disciplinas educativas estudiadas fueron los cuestionarios $(29,4 \%)$ y los registros de juego $(25,9 \%)$. Por otro lado, los menos empleados fueron los grupos de reflexión $(5,9 \%)$ y las entrevistas $(8,2 \%)$. Se destaca el uso de diarios de observación en Lingüística (30 \%), de encuestas en Ciencias Naturales (38,46 \%), de test de evaluación en Matemáticas, Física y Química (30 \%) y en Ciencias Sociales (27,27 \%), y de grupos de reflexión en Educación Física (26,67 \%).

Los procedimientos de evaluación empleados en mayor medida han sido experimentales más que observacionales, no obstante, hay que contar con las particularidades de cada disciplina educativa. En este sentido, en un contexto digital que ha sido gamificado, según Santiago y Bergmann (2018), los docentes deben proporcionar al alumnado formas diferentes de evaluación formativa. Por tanto, tiene sentido ajustar los esfuerzos de gamificación en un contexto digital mientras el alumnado practica ejercicios, usando, por ejemplo, un portafolio o registro digital de aprendizaje y, por tanto, usándolo como instrumento para la evaluación formativa, lo que permitirá al alumnado su propio aprendizaje, y en los docentes, a mejorar la adquisición de información, datos y resultados de aprendizaje. Esta estrategia suscita el uso de herramientas digitales en la evaluación, con la finalidad 
JOEL MANUEL PRIETO ANDREU

REVISIÓN SISTEMÁTICA SOBRE LA EVALUACIÓN DE PROPUESTAS DE GAMIFICACIÓN EN SIETE DISCIPLINAS EDUCATIVAS

de mejorar el compromiso del alumnado en el proceso de evaluación (Holguín et al., 2020). Hay numerosos estudios que se han servido de alguna herramienta digital de evaluación, según Rodríguez et al. (2019) se corrobora que la metodología planteada basada en la herramienta "Quizizz" ayuda a que se sientan seguros de sí mismos para enfrentarse a un examen tipo test, favoreciendo el trabajo en equipo y siendo motivadora y eficaz. Por otro lado, "Qstream" es una herramienta considerada tanto como una red social de aprendizaje como un sistema de evaluación colaborativa (peer-review) o personalizada, permitiendo su uso bajo soporte PC o móvil/tablet.

Por otra parte, kahoot!, a pesar de ser un juego diseñado con fines educativos, es tan divertido y entretenido como los diseñados específicamente con una finalidad lúdica, además de producir un aumento de la tasa de éxito y de rendimiento en la asignatura (Parejo-Moruno et al., 2018). En la misma línea, Conde de Felipe (2020) también empleó Kahoot para convertir una prueba de evaluación en un juego de competición entre los estudiantes. No obstante, el factor novedoso, causante en gran parte del aumento de la motivación de los estudiantes tiende a ir reduciendo, hasta llegar a desaparecer a los 4 años aproximadamente (Wang, 2015).

En cuanto a los resultados motivacionales y de aprendizaje de los artículos incluidos en la revisión, las variables psicológicas que más se evaluaron en las propuestas gamificadas fueron el aprendizaje (47,1\%) y la motivación (40 \%), siendo todos los resultados positivos. Se destaca la evaluación del disfrute en Educación Física (9,09 \%), del rendimiento en Fisioterapia y Nutrición (13,33 \%) y de la satisfacción en Informática (8,70 \%). En Simons et al. (2021) implantaron en el aula un juego serio que mejoró el rendimiento, destacando que tenían más habilidades los que tenían una mayor experiencia en el entorno digital y en videojuegos. El modelo TETEM propone dos características clave que pueden afectar a la eficacia de la experiencia ludificada en el sujeto: la actitud y la experiencia con los videojuegos, puesto que supuestamente predicen la reacción del sujeto a la experiencia gamificada.

Por otra parte, existe algún estudio como el de Cameron y Bizo (2019) que muestra resultados contradictorios respecto a la mayor parte del cuerpo base del estudio, señalando que la herramienta utilizada (Kaboot!') no aumentó directamente el rendimiento; en Lobet et al. (2021) se señala como punto negativo la duración excesiva que poseía el videojuego pedagógico, aunque se mejoró la motivación del alumnado con su implantación; en Karaaslan (2018) no se produjo aprendizaje porque a los estudiantes no les gustaban los juegos digitales, los consideraban innecesarios o infantiles, no encontrando nada curioso en ellos o porque no sentían atracción por el contenido. En estos casos, se podría recomendar que el alumnado elabore sus propios juegos siguiendo las instrucciones del profesorado o modificarlos según los comentarios constructivos más frecuentes de los estudiantes.; o en el estudio de Zhang y Chen (2021) en el que se evaluó la ansiedad del alumnado ante un proceso de gamificación, los estudiantes que se sintieron más ansiosos debido a su personalidad introvertida o falta de preparación, el efecto de la gamificación en la reducción de la ansiedad fue menos obvio. 


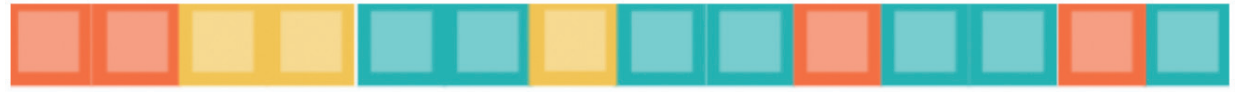

.. Instrumentos de eualuación y su influencia en los procesos de gamificación .:.

PROCEDIMIENTOS EXPERIMENTALES

CUESTIONARIOS

- $(p=015)$

MOTIUACIÓN
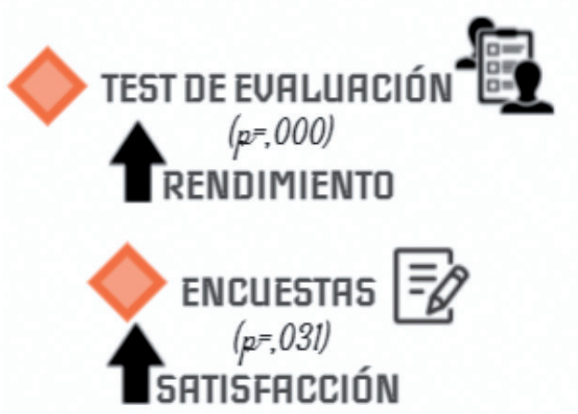

PROCEDIMIENTOS OBSERVACIONALES

GRUPOS DE REFLEXIÓN UPARTICIPACIÓN

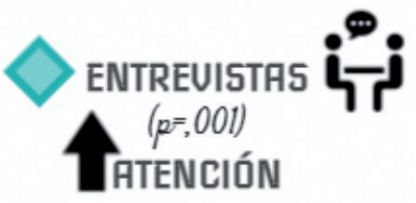

Fuente: Elaboración propia

En el área temática de gamificación educativa, se hace necesaria la contribución científica en relación con el trabajo de diversas variables psicológicas menos estudiadas como la actitud, el compromiso, el estrés, la ansiedad y el rendimiento en propuestas didácticas gamificadas. Por otro lado, se establecieron correlaciones significativas entre los grupos de reflexión con una mejora en la participación, el uso de cuestionarios en la mejora de la motivación, entrevistas para mejorar la atención, el uso de test de evaluación para mejorar el rendimiento, y encuestas para mejorar la satisfacción.

Se señala que las mencionadas combinaciones arrojaron correlaciones significativas, siendo recomendable priorizar el uso de dichos instrumentos de evaluación 
JOEL MANUEL PRIETO ANDREU

REVISIÓN SISTEMÁTICA SOBRE LA EVALUACIÓN DE PROPUESTAS DE GAMIFICACIÓN EN SIETE DISCIPLINAS EDUCATIVAS

si se tiene como objetivo mejorar alguna de las variables psicológicas que se han relacionado de forma positiva con dichos instrumentos de evaluación en cualquier proceso de gamificación (Figura 6).

En el estudio de Núñez et al. (2021) concluyeron que a medida que se incluyen más mecánicas y dinámicas en una propuesta gamificada se mejora la evaluación formativa en el contexto digital, y más cambia significativamente el aprendizaje de las matemáticas. La experiencia llevada a cabo puso de manifiesto la ambigüedad del posicionamiento de la motivación en el juego entre la gamificación y la evaluación formativa empleada en dicho estudio por medio de la herramienta digital gamificada llamada retomates.

En la presente revisión sistemática, las principales variables psicológicas estudiadas fueron la motivación y el aprendizaje, echando en falta una mayor profundización en otras variables como el compromiso, el rendimiento, o la atención, entre otras. Por otro lado, se debería profundizar en la comprensión de los efectos de las recompensas extrínsecas a lo largo del tiempo. Por último, la gamificación bajo un soporte digital tiene un enorme potencial en el uso de herramientas de evaluación específicas, como puntos de seguimiento en tiempo real, tablas de clasificación, o cualquier registro de datos de juego en Internet. Las aplicaciones gamificadas podrían ayudar a estimular la motivación intrínseca de los estudiantes al promover su iniciativa para hacer la actividad por sí misma, por la diversión y por la satisfacción que experimentan mientras aprenden. Tras esta revisión, se puede afirmar que la gamificación es una metodología de enseñanza eficaz por la que merece la pena invertir en programas de formación para el profesorado. Sin embargo, no se han realizado esfuerzos para medir el grado de aprendizaje o rendimiento en un proceso educativo gamificado bajo algún instrumento validado. En este sentido, Kalogiannakis et al. (2021) señalan que la implementación en diferentes niveles educativos, la falta de herramientas de evaluación específicas y el uso de elementos de juego específicos, arrojan resultados contradictorios. Por esta razón, se echa en falta la validación de herramientas de evaluación específicas o una normalización de pautas específicas como las propuestas en Flores (2019) para llevar a buen término cualquier proceso de gamificación en el aula.

\section{REFERENCIAS BIBLIOGRÁFICAS}

Alshammari, M. T. (2020). Evaluation of Gamification in E-Learning Systems for Elementary School Students. TEM Journal, 9(2), 806-813. https://doi.org/10.18421/TEM92-51

Cameron, K., \& Bizo, L. A. (2019). Use of the game-based learning platform KAHOOT! to facilitate learner engagement in Animal Science students. Research in Learning Technology, 27, 1-14. https://doi.org/10.25304/rlt.v27.2225

Colomo-Magaña, E., Sánchez-Rivas, E., Ruiz-Palmero, J., y Sánchez-Rodríguez, J. (2020). Percepción docente sobre la gamificación de la evaluación en la asignatura de Historia en educación secundaria. Información tecnológica, 31(4), 233-242. https://doi.org/10.4067/ S0718-07642020000400233 
Conde de Felipe, M. M., Molina Caballero, J. M., y Ruiz Reyes, A. (2020). Evaluación puntual del aprendizaje mediante una técnica de gamificación. VII Jornadas Iberoamericanas de Innovación Educativa en el Ámbito de las TIC y las TAC. Universidad de Las Palmas de Gran Canaria (ULPGC), 395-401.

Flores, G. (2019). jJugamos al Súper Mario Bros? Descripción de una experiencia gamificada en la formación del profesorado de Educación Física. Retos, 36, 529-534.

Hernando, M., González, C., Zurita, C., Almirell, L., y Guitert, M. (2015). Play the Game: ludificació i hàbits saludables en l'educació física. Apunts Educació Física i Esports, 119, 71-79. https://doi.org/10.5672/apunts.2014-0983.es.(2015/1).119.04

Holguín, F., Holguín, E., y García, N. (2020). Gamificación de la enseñanza de las matemáticas: una revisión sistemática. Telos: revista de Estudios Interdisciplinarios en Ciencias Sociales, 22(1), 62-75. https://doi.org/10.36390/telos221.05

Kalogiannakis, M., Papadakis, S., \& Zourmpakis, A. I. (2021). Gamification in science education. A systematic review of the literature. Education Sciences, 11(22),2-36. https://doi. org/10.3390/educsci11010022

Karaaslan, H., Kilic, N., Guven-Yalcin, G., \& Gullu, A. (2018). Students' reflections on vocabulary learning through synchronous and asynchronous games and activities. Turkish Online Journal of Distance Education, 19(3), 53-70.

Leitão, R., Maguire, M., Turner, S., \& Guimarães, L. (2021). A systematic evaluation of game elements effects on students' motivation. Education and Information Technologies, 1-23. https://doi.org/10.1007/s10639-021-10651-8

Ling, L. T. Y. (2018). Meaningful Gamification and Students' Motivation: A Strategy for Scaffolding Reading Material. Online Learning, 22(2), 141-155. https://doi.org/10.24059/olj.v22i2.1167

Lobet, G., Descamps, C., Leveau, L., Guillet, A., \& Rees, J. F. (2021). QuoVidi: An open-source web application for the organization of large-scale biological treasure hunts. Ecology and evolution, 11(8), 3516-3526. https://doi.org/10.1002/ece3.7130

López, E. L., Tobón, S., y Hernández, L. G. J. (2019). Escala para evaluar artículos científicos en ciencias sociales y humanas-EACSH. REICE: Revista Iberoamericana Sobre Calidad, Eficacia y Cambio En Educación, 17(4), 111-125. https://doi.org/10.15366/reice2019.17.4.006

Moher, D., Liberati, A., Tetzlaff, J., \& Altman, D. G., The PRISMA Group (2009). Preferred Reporting Items for Systematic Reviews and Meta-Analyses: The PRISMA Statement. PLoS Med 6(6): e1000097. https://doi.org/10.1371/journal.pmed1000097

Mora-González, J., Pérez-López, I. J., \& Delgado-Fernández, M. (2020). The «\$ in TIME» gamification project: using a mobile app to improve cardiorespiratory fitness levels of college students. Games for health journal, 9(1), 37-44. https://doi.org/10.1089/g4h.2019.0001

Miralles, P. y Monteagudo, J. (2019). Métodos, instrumentos y procedimientos para conocer cómo se evalúan las competencias históricas. Educar em revista, 35(74), 127-144. https:// doi.org/10.1590/0104-4060.64404

Nair, S., \& Mathew, J. (2021). Evaluation of a Gamified Learning Experience: Analysis of the impact of gamification on learning outcomes in education. Revista Conbecimento Online, 2, 4-20. https://doi.org/10.25112/rco.v2i0.2518

Núñez, R. P., Suárez, C. A. H., y Castro, W. R. A. (2021). Gamificación y evaluación formativa en la asignatura de matemática a través de herramienta web 2.0. Revista Boletín Redipe, 10(7), 243-261. https://doi.org/10.36260/rbr.v10i7.1361 
JOEL MANUEL PRIETO ANDREU

REVISIÓN SISTEMÁTICA SOBRE LA EVALUACIÓN DE PROPUESTAS DE GAMIFICACIÓN EN SIETE DISCIPLINAS EDUCATIVAS

Parejo-Moruno, F. M., Linares-Luján, A. M., Vega-Gómez, F. I., y Preciado, J. F. R. (2018). El uso de herramientas de gamificación como mecanismo de control y evaluación en la realización de lecturas. Investigaciones en historia económica: su transferencia a la docencia, 414-436.

Pérez-López, I. J.; Rivera García, E., y Trigueros Cervantes, C. (2017). «La profecía de los elegidos»: un ejemplo de gamificación aplicado a la docencia universitaria. Revista Internacional de Medicina y Ciencias de la Actividad Física y el Deporte, 17(66), 243-260. https://doi.org/10.15366/rimcafd2017.66.003

Prieto, J. M. (2020). Una revisión sistemática sobre gamificación, motivación y aprendizaje en universitarios. Teoría De La Educación. Revista Interuniversitaria, 32(1), 73-99. https:// doi.org/10.14201/teri.20625

Quintero, L. E., Jiménez, F., y Area, M. (2018). Más allá del libro de texto. La gamificación mediada con TIC como alternativa de innovación en Educación Física. Retos, 34, 343-348. https://doi.org/10.47197/retos.v0i34.65514

Rodríguez, D. V., Mezquita, J. M. M., y Vallecillo, A. I. G. (2019). Metodología innovadora basada en la gamificación educativa: evaluación tipo test con la herramienta Quizizz. Profesorado, Revista de Currículum y Formación del Profesorado, 23(3), 363-387. https:// doi.org/10.30827/profesorado.v23i3.11232

Rodríguez-Oroz, D., Gómez-Espina, R., Bravo Pérez, M. J., y Truyol, M. E. (2019). Aprendizaje basado en un proyecto de gamificación: vinculando la educación universitaria con la divulgación de la geomorfología de Chile. Revista Eureka sobre Enseñanza y Divulgación de las Ciencias 16(2). https://doi.org/10.25267/RevEurekaensendivulgcienc.2019. v16.i2.2202

Santiago, R., y Bergmann, J. (2018) Aprender al revés. Flipped learning 3.0 y metodologías activas en el aula. Paidós.

Sevilla Pavón, A., \& Haba Osca, J. (2017). Learning from real life and not books: A gamified approach to task design in transatlantic telecollaboration. Iberica, 33, 235-260. https:// www.aelfe.org/documents/33_10_IBERICA.pdf

Simons, A., Wohlgenannt, I., Weinmann, M., \& Fleischer, S. (2021). Good gamers, good managers? A proof-of-concept study with Sid Meier's civilization. Review of Managerial Science, 15(4), 957-990. https://doi.org/10.1007/s11846-020-00378-0

Swacha, J. (2021). State of research on gamification in education: A bibliometric survey. Education Sciences, 11(2), 69. 9. https://doi.org/10.3390/educsci1102006

Ucar, H., \& Kumtepe, A.T. (2017). Using the game-based student response tool kahoot! In an online class: perspectives of online learners. En Resta y Smith (Eds.), Proceedings of Society for Information Technology \& Teacher Education International Conference (pp. 303-307). Association for the Advancement of Computing in Education (AACE).

Wang, A. I. (2015). The wear out effect of a game-based student response system. Computers and Education, 82, 217-227. https://doi.org/10.1016/j.compedu.2014.11.004

Zhang, L., \& Chen, Y. (2021). Examining the Effects of Gamification on Chinese College Students' Foreign Language Anxiety: A Preliminary Exploration. En 4th International Conference on Big Data and Education (pp. 1-5). https://doi.org/10.1145/3451400.3451401 
JOEL MANUEL PRIETO ANDREU

REVISIÓN SISTEMÁTICA SOBRE LA EVALUACIÓN DE PROPUESTAS DE GAMIFICACIÓN

EN SIETE DISCIPLINAS EDUCATIVAS

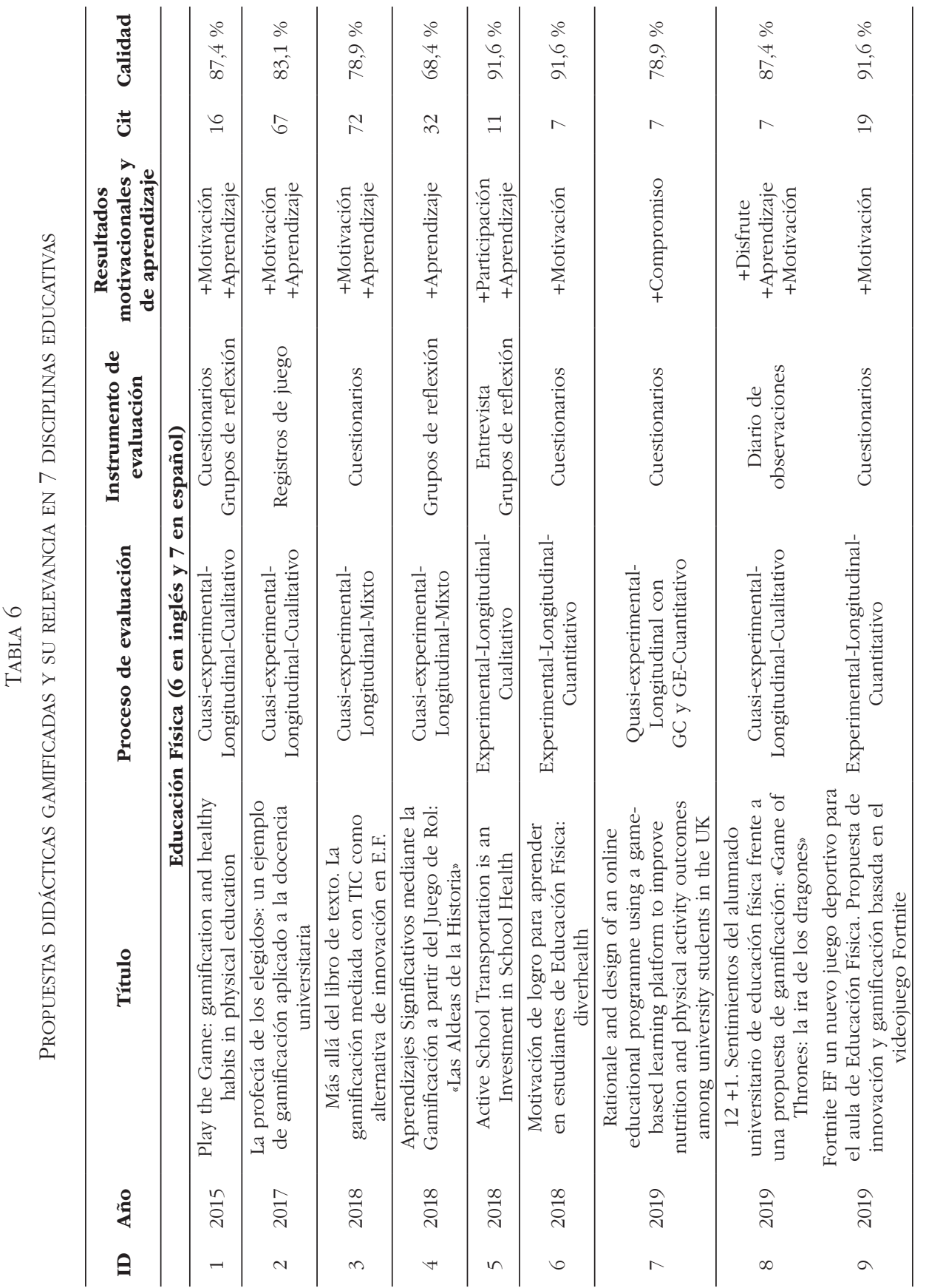



EN SIETE DISCIPLINAS EDUCATIVAS

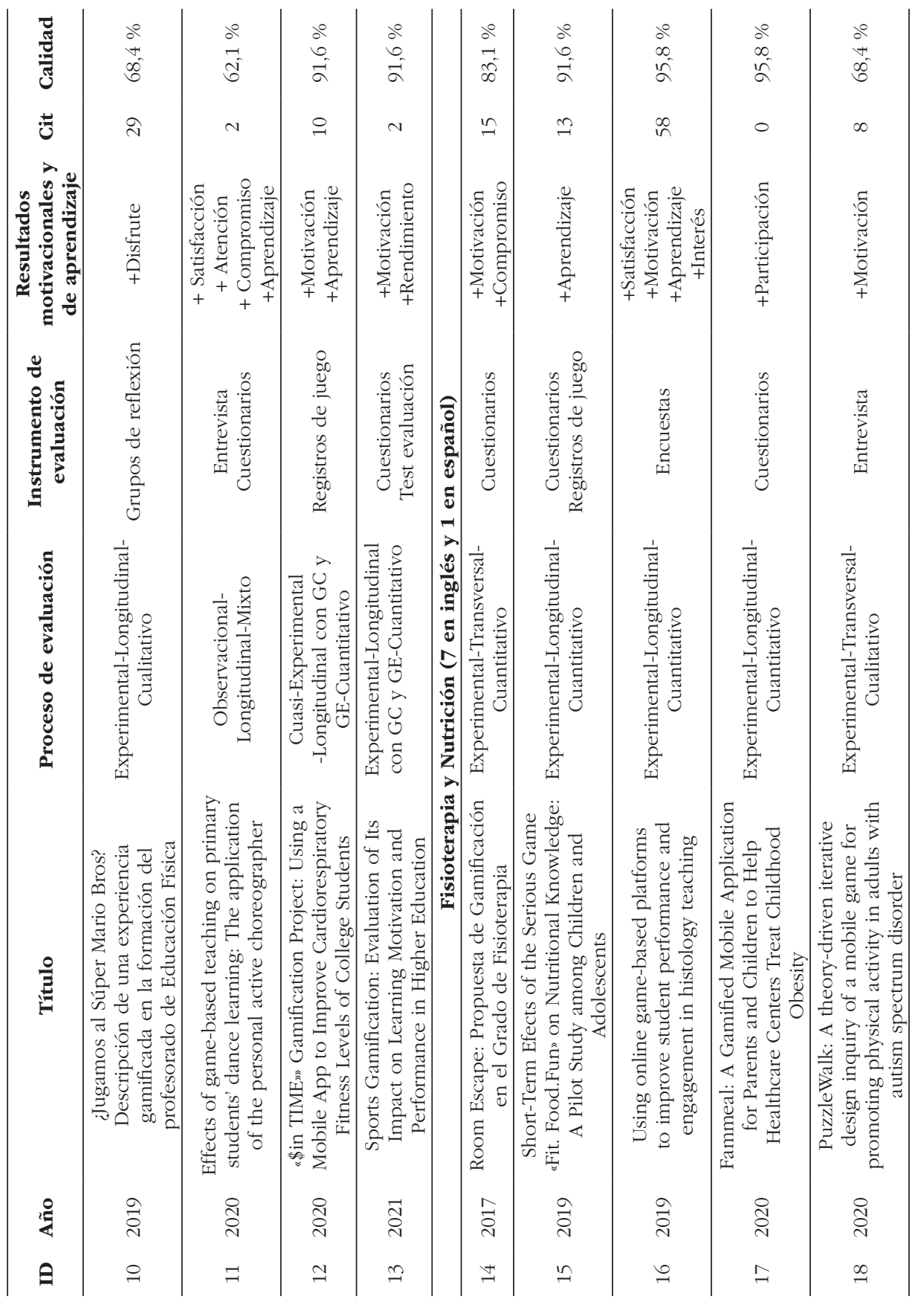


JOEL MANUEL PRIETO ANDREU

209

REVISIÓN SISTEMÁTICA SOBRE LA EVALUACIÓN DE PROPUESTAS DE GAMIFICACIÓN

EN SIETE DISCIPLINAS EDUCATIVAS

\begin{tabular}{|c|c|c|c|c|c|c|c|c|c|c|c|c|}
\hline 苞 & $\begin{array}{l}\stackrel{\circ}{\circ} \\
\dot{+} \\
\infty\end{array}$ & $\begin{array}{l}\stackrel{0}{0} \\
\text { i } \\
\text { - }\end{array}$ & $\begin{array}{l}d^{\circ} \\
+ \\
\frac{\infty}{n}\end{array}$ & & $\begin{array}{l}\stackrel{0}{0} \\
\dot{\sigma} \\
i\end{array}$ & $\begin{array}{l}\stackrel{0}{2} \\
\stackrel{0}{\circ}\end{array}$ & $\begin{array}{l}{ }^{0} \\
\stackrel{2}{0} \\
\infty^{\circ}\end{array}$ & $\begin{array}{l}\stackrel{0}{0} \\
\stackrel{\infty}{\infty}\end{array}$ & $\begin{array}{l}\partial^{0} \\
\otimes_{0}^{\prime} \\
\infty_{0}^{\circ}\end{array}$ & $\begin{array}{l}\stackrel{0}{2} \\
\hat{n} \\
\text { in }\end{array}$ & $\begin{array}{l}\partial^{\circ} \\
\hat{n} \\
i n\end{array}$ & $\begin{array}{l}\stackrel{0}{0} \\
\stackrel{1}{0} \\
\infty_{0}^{\circ}\end{array}$ \\
\hline : & $\infty$ & 0 & N & & $\stackrel{\infty}{\sim}$ & શે & N & 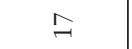 & - & N & - & \\
\hline 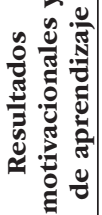 & 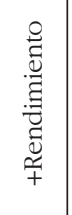 & 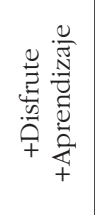 & 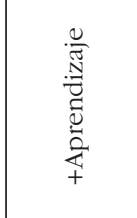 & & & 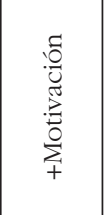 & 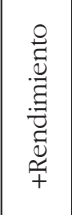 & $\begin{array}{l}0 \\
. \\
0 \\
0 \\
\tilde{\Xi} \\
00 \\
+ \\
+\end{array}$ & 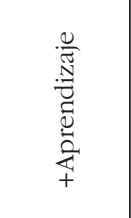 & 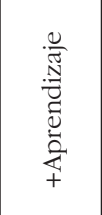 & 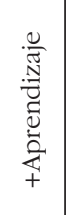 & 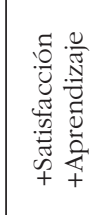 \\
\hline 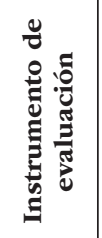 & 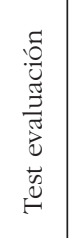 & 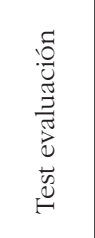 & 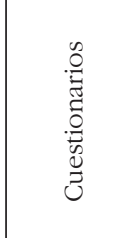 & 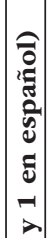 & 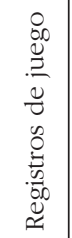 & 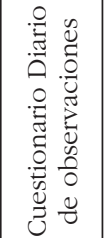 & 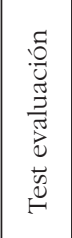 & 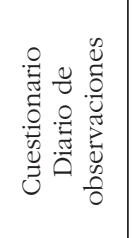 & 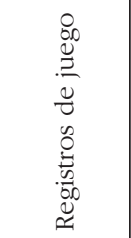 & 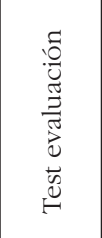 & 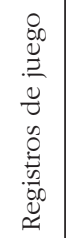 & 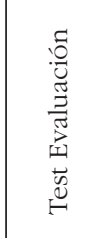 \\
\hline 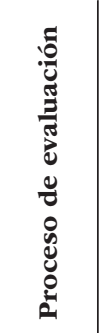 & 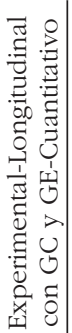 & 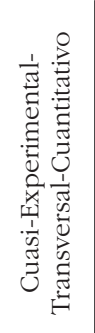 & 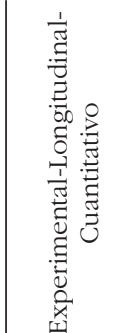 & 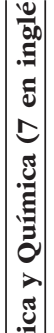 & 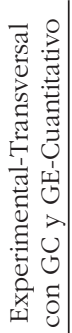 & 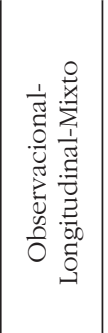 & 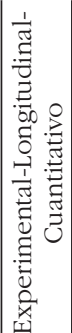 & 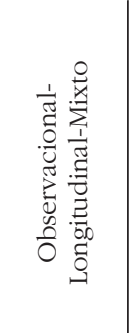 & 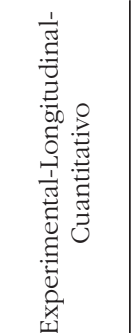 & 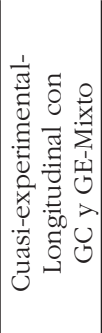 & 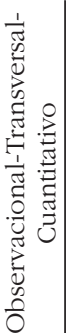 & 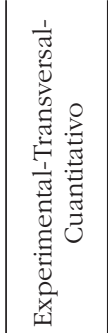 \\
\hline$\stackrel{\circ}{Z}$ & 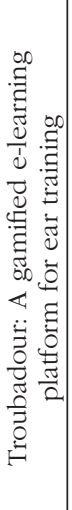 & 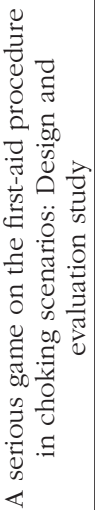 & 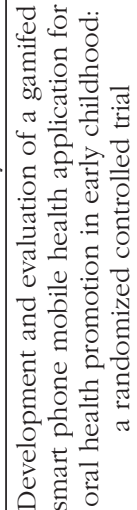 & 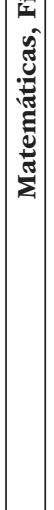 & 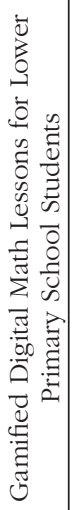 & 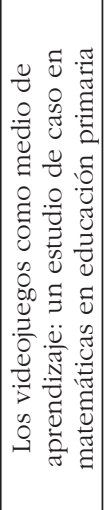 & 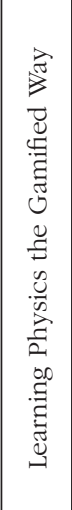 & 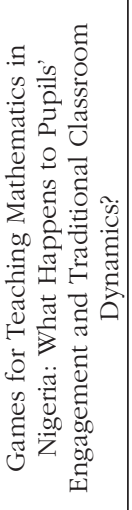 & 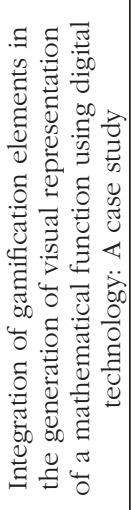 & 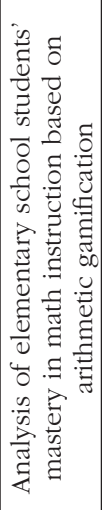 & 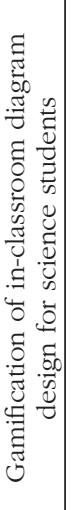 & 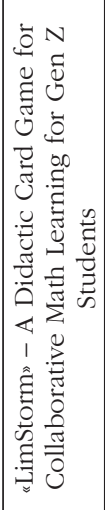 \\
\hline 宅 & $\begin{array}{l}\text { ָे } \\
\text { (2) }\end{array}$ & ঙे & त्ञ & & $\stackrel{\vec{\Xi}}{\vec{i}}$ & 竞 & 竝 & $\overrightarrow{\vec{~}}$ & $\overrightarrow{\vec{~}}$ & $\stackrel{\vec{े}}{\vec{i}}$ & हें & त्ञ \\
\hline 列 & ? & i & $\vec{v}$ & & $\tilde{\lambda}$ & $\widetilde{\sim}$ & $\stackrel{+}{N}$ & $\stackrel{n}{v}$ & ำ & $\grave{v}$ & $\stackrel{\sim}{\sim}$ & \\
\hline
\end{tabular}



EN SIETE DISCIPLINAS EDUCATIVAS

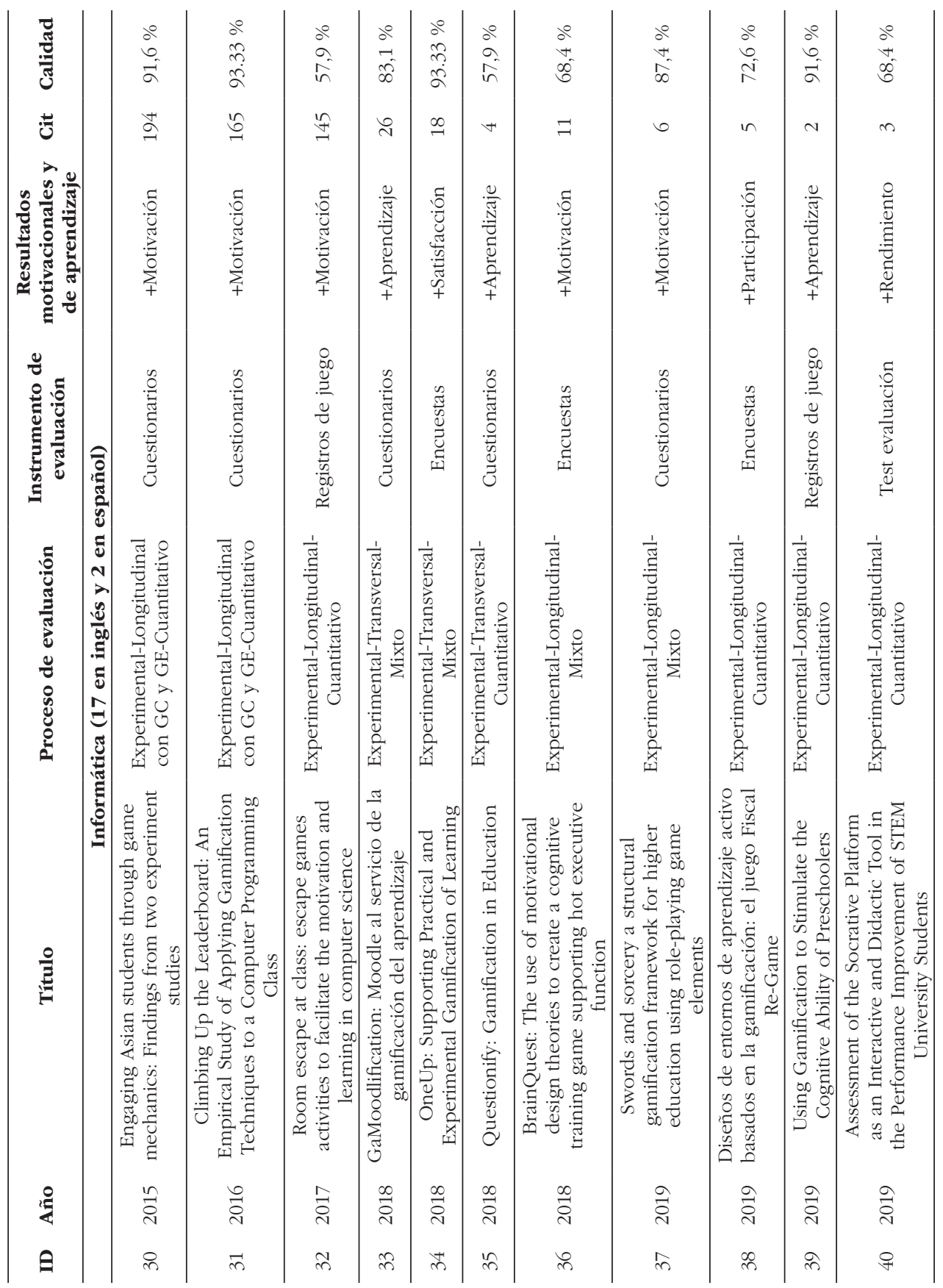


JOEL MANUEL PRIETO ANDREU

REVISIÓN SISTEMÁTICA SOBRE LA EVALUACIÓN DE PROPUESTAS DE GAMIFICACIÓN

EN SIETE DISCIPLINAS EDUCATIVAS

\begin{tabular}{|c|c|c|c|c|c|c|c|c|c|c|c|c|c|}
\hline & $\begin{array}{l}\text { do } \\
\text { in }\end{array}$ & $\begin{array}{l}\partial^{\circ} \\
\text { ñ } \\
\text { nn }\end{array}$ & $\begin{array}{l}\alpha^{2} \\
\infty \\
\swarrow \\
\alpha\end{array}$ & $\begin{array}{l}\text { do } \\
\infty \\
\alpha \\
\alpha\end{array}$ & $\frac{d}{\dot{*}}$ & $\begin{array}{l}\dot{a} \\
\vdots \\
\vec{\sigma}\end{array}$ & $\begin{array}{l}\dot{0} \\
b \\
i \\
-1\end{array}$ & $\begin{array}{l}\text { dᄋ } \\
\overrightarrow{3} \\
\text { ji }\end{array}$ & & $\begin{array}{l}\partial^{2} \\
\text { in }\end{array}$ & $\begin{array}{l}\alpha^{\circ} \\
\infty \\
\end{array}$ & $\begin{array}{l}\stackrel{0}{0} \\
\sigma \\
-1\end{array}$ & $\begin{array}{l}\text { o̊ } \\
\dot{0} \\
i\end{array}$ \\
\hline نㅊ & 우 & $n$ & in & $n$ & 6 & $\infty$ & 0 & 0 & & $\exists$ & $n$ & $\stackrel{\tilde{r}}{=}$ & $\stackrel{ }{-}$ \\
\hline 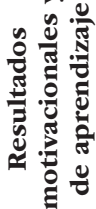 & 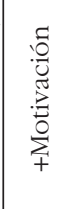 & 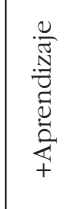 & 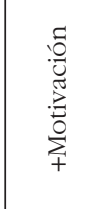 & 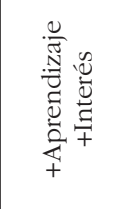 & 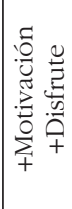 & 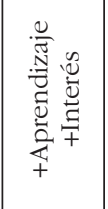 & 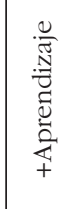 & 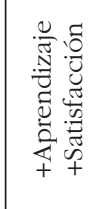 & & 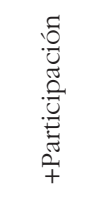 & 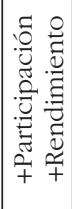 & 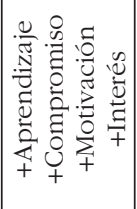 & $\begin{array}{l}0 \\
.0 \\
.0 \\
0 \\
\tilde{\Xi} \\
0 \\
0 \\
+\end{array}$ \\
\hline 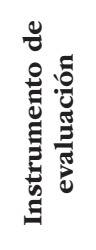 & $\begin{array}{l}0 \\
80 \\
0 \\
.0 \\
0 \\
0 \\
0 \\
0 \\
0 \\
00 \\
0 \\
0 \\
0\end{array}$ & 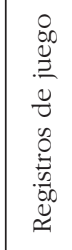 & 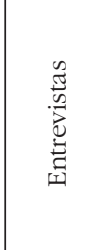 & 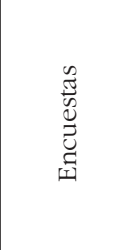 & 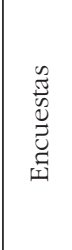 & 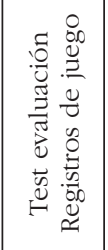 & 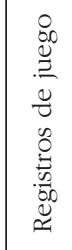 & 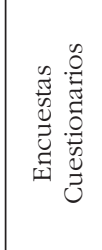 & 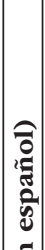 & 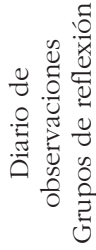 & 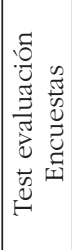 & 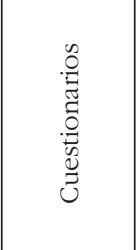 & 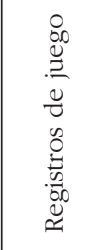 \\
\hline 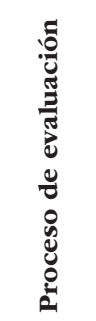 & 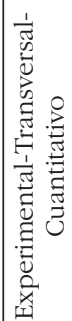 & 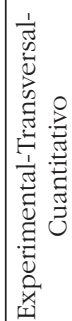 & 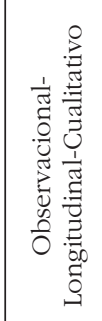 & 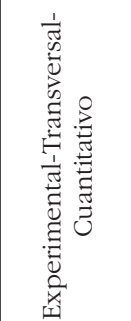 & 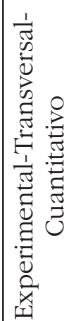 & 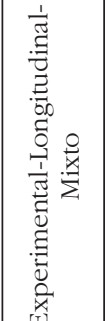 & 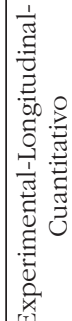 & 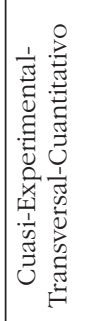 & 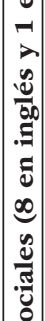 & 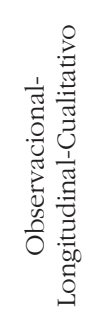 & 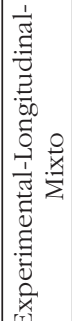 & 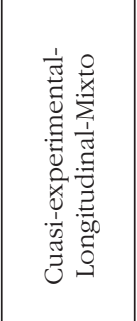 & 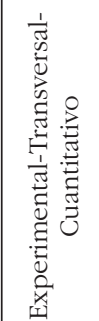 \\
\hline 象 & 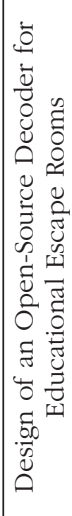 & 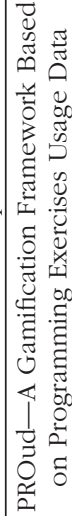 & 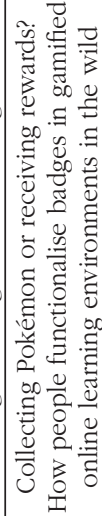 & 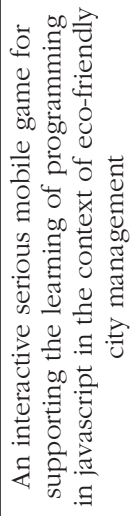 & 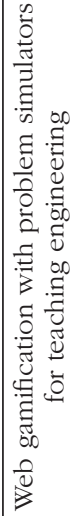 & 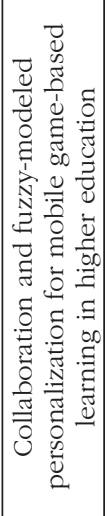 & 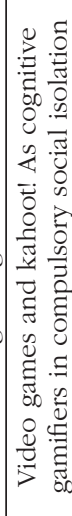 & 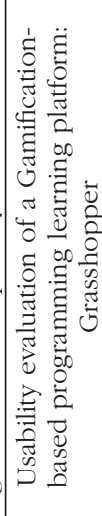 & كَّ & 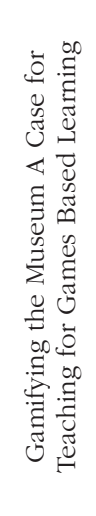 & 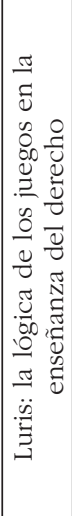 & 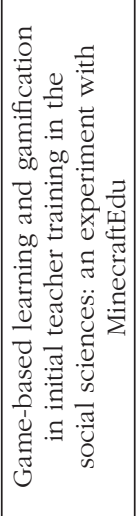 & 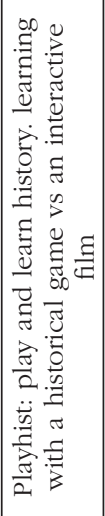 \\
\hline 安 & $\overrightarrow{\vec{D}}$ & $\overrightarrow{\vec{\sigma}}$ & $\overrightarrow{\vec{\sigma}}$ & ঙ્ડ & त్ & ָั & ્ֻণิ & त् & & $\stackrel{n}{\tilde{N}}$ & 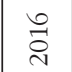 & $\stackrel{0}{\stackrel{\sim}{*}}$ & $\overrightarrow{\vec{i}}$ \\
\hline 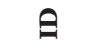 & $\vec{F}$ & $\underset{f}{ }$ & $\stackrel{\sim}{F}$ & $\underset{t}{F}$ & $\stackrel{i f}{f}$ & 아 & $\stackrel{F}{F}$ & Fै & & $\stackrel{F}{ }$ & in & $\vec{n}$ & in \\
\hline
\end{tabular}



EN SIETE DISCIPLINAS EDUCATIVAS

\begin{tabular}{|c|c|c|c|c|c|c|c|c|c|c|c|c|}
\hline : & $\begin{array}{l}\text { वீ } \\
\text { तु }\end{array}$ & $\begin{array}{l}\stackrel{0}{ } \\
\text { in } \\
\text { in }\end{array}$ & $\begin{array}{l}\dot{o}^{2} \\
+ \\
\frac{\infty}{n}\end{array}$ & $\begin{array}{l}\text { do } \\
\text { b } \\
i \\
i\end{array}$ & $\begin{array}{l}{ }_{0}^{\circ} \\
\text { ñ } \\
\text { in }\end{array}$ & & $\begin{array}{l}\stackrel{0}{2} \\
\stackrel{2}{n}\end{array}$ & $\begin{array}{l}\stackrel{0}{2} \\
\text { in }\end{array}$ & $\begin{array}{l}\text { do } \\
b \\
\vec{a}\end{array}$ & $\begin{array}{l}\stackrel{0}{0} \\
\vec{\infty}\end{array}$ & $\begin{array}{l}\text { do } \\
b \\
\vec{a}\end{array}$ & $\begin{array}{l}\alpha^{2} \\
\infty \\
\kappa\end{array}$ \\
\hline$:$ & $\curvearrowright$ & $N$ & 0 & $\infty$ & 0 & & $N$ & 0 & $N$ & 6 & $\infty$ & \\
\hline 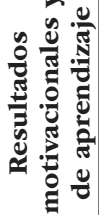 & 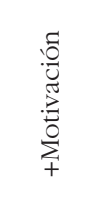 & 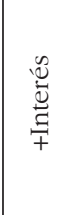 & 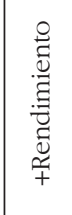 & 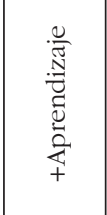 & 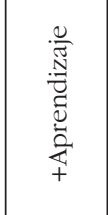 & & 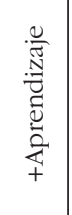 & 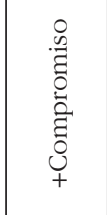 & $\begin{array}{l}0 \\
.0 \\
0 \\
0 \\
\tilde{\Xi} \\
0 \\
0 \\
+\end{array}$ & 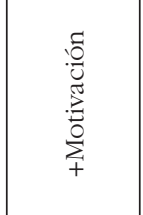 & 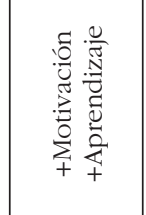 & 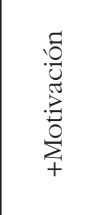 \\
\hline 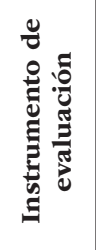 & 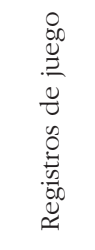 & 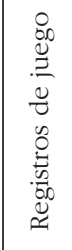 & 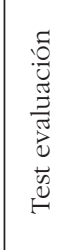 & 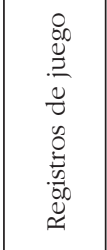 & 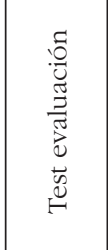 & 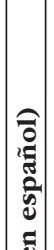 & 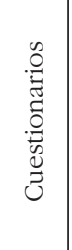 & 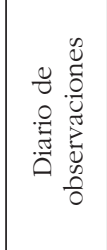 & 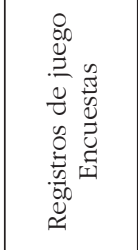 & 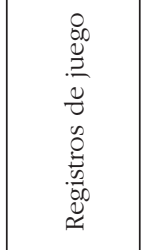 & 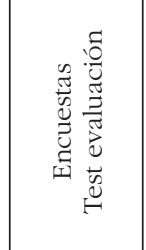 & 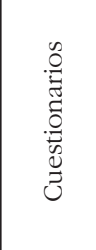 \\
\hline 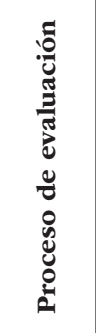 & 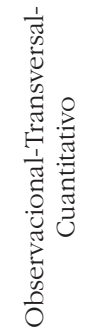 & 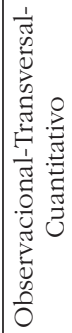 & 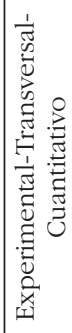 & 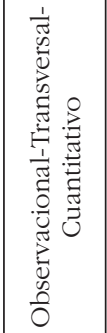 & 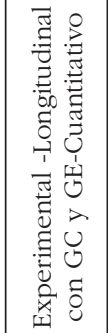 & 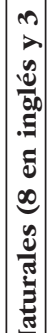 & 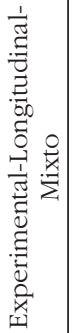 & 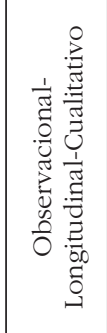 & 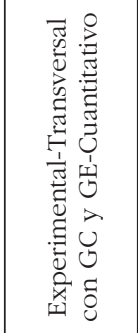 & 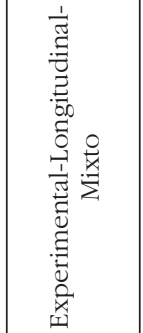 & 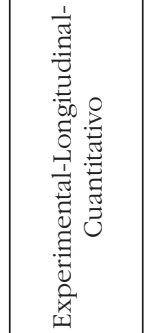 & 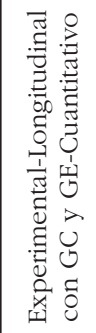 \\
\hline$\stackrel{0}{\vec{E}}$ & 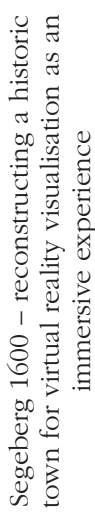 & 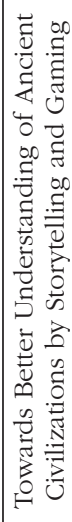 & 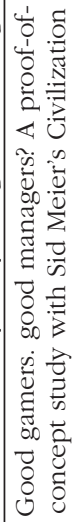 & 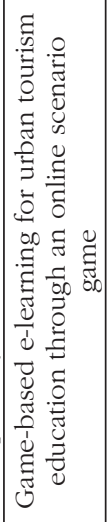 & 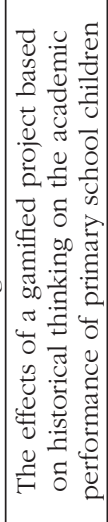 & 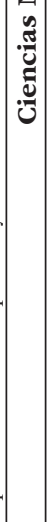 & 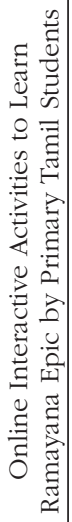 & 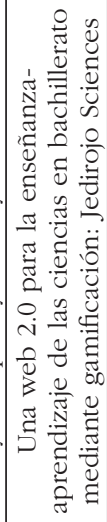 & 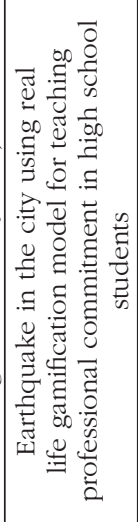 & 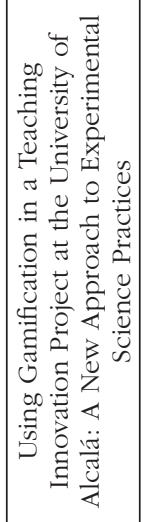 & 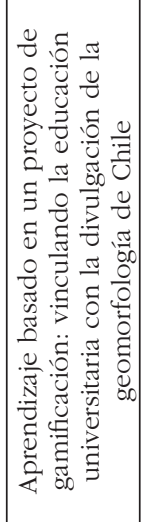 & 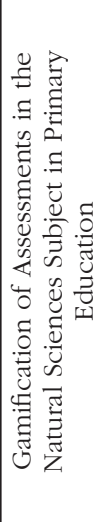 \\
\hline 安 & $\vec{~} \vec{d}$ & $\stackrel{\infty}{\stackrel{\infty}{\sim}}$ & ્ֻડి & ્ָ & त్ & & $\begin{array}{l}\stackrel{0}{c} \\
\stackrel{\sim}{n}\end{array}$ & $\stackrel{\infty}{\stackrel{\infty}{\sim}}$ & $\stackrel{\infty}{\stackrel{\infty}{二}}$ & $\stackrel{\vec{\nu}}{\vec{\nu}}$ & $\stackrel{\overrightarrow{\mathrm{d}}}{ }$ & $\Rightarrow$ \\
\hline 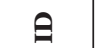 & & in & in & $\stackrel{\imath}{\Perp}$ & & & $\stackrel{\infty}{n}$ & in & & & & \\
\hline
\end{tabular}


JOEL MANUEL PRIETO ANDREU

REVISIÓN SISTEMÁTICA SOBRE LA EVALUACIÓN DE PROPUESTAS DE GAMIFICACIÓN

EN SIETE DISCIPLINAS EDUCATIVAS

\begin{tabular}{|c|c|c|c|c|c|c|c|c|c|c|c|c|}
\hline 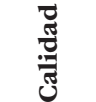 & $\begin{array}{l}\frac{0}{2} \\
\frac{1}{\infty} \\
\infty\end{array}$ & $\begin{array}{l}\dot{0} \\
\dot{b} \\
i\end{array}$ & $\begin{array}{l}\dot{0}^{\circ} \\
\stackrel{0}{1} \\
\infty^{0}\end{array}$ & $\begin{array}{l}d^{\circ} \\
t_{n} \\
\infty\end{array}$ & $\begin{array}{l}\stackrel{0}{ } \\
\stackrel{2}{\infty} \\
\infty^{2}\end{array}$ & & $\begin{array}{l}\text { o̊ } \\
\text { in }\end{array}$ & $\begin{array}{l}\stackrel{0}{0} \\
\vec{j}\end{array}$ & $\begin{array}{l}\dot{0}^{2} \\
\hat{n} \\
i n\end{array}$ & $\begin{array}{l}\dot{0}^{2} \\
\hat{n}\end{array}$ & $\begin{array}{l}\text { of } \\
\dot{t} \\
\infty\end{array}$ & $\begin{array}{l}\dot{0}^{0} \\
\vec{\infty} \\
\infty\end{array}$ \\
\hline : & $\stackrel{\sim}{\sim}$ & in & 0 & $\tau$ & 0 & & $\stackrel{\infty}{\sim}$ & $\ddot{\sim}$ & $\infty$ & $\stackrel{\infty}{\sim}$ & $\triangleq$ & $\underset{\forall}{*}$ \\
\hline 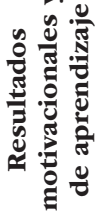 & 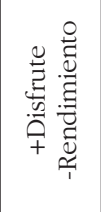 & 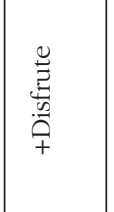 & 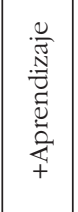 & 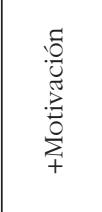 & 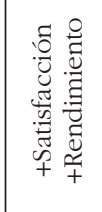 & & 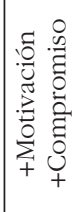 & 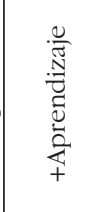 & 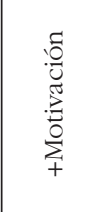 & 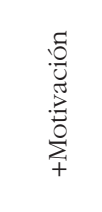 & 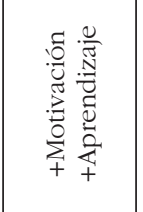 & 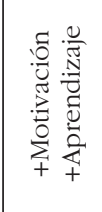 \\
\hline 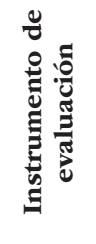 & 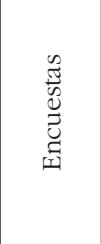 & 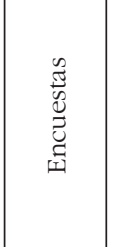 & 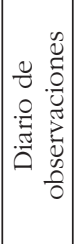 & 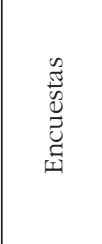 & 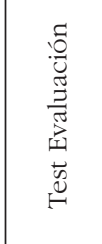 & 흥 & 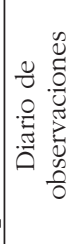 & 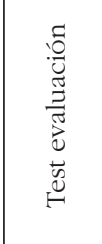 & 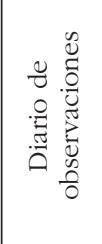 & 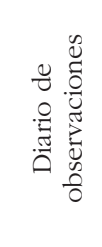 & 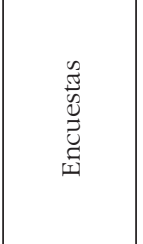 & 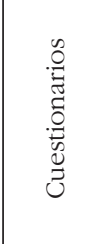 \\
\hline 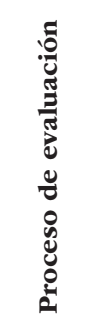 & 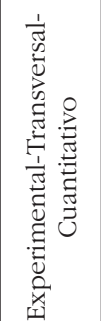 & 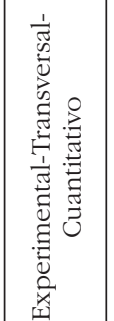 & 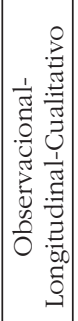 & 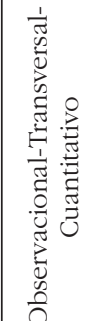 & 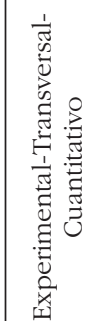 & 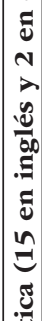 & 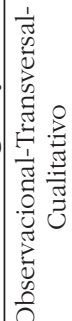 & 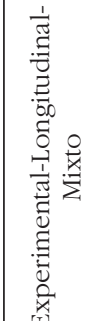 & 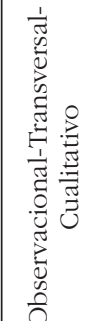 & 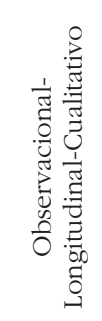 & 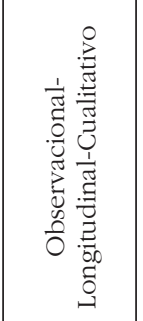 & 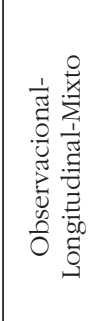 \\
\hline 总 & 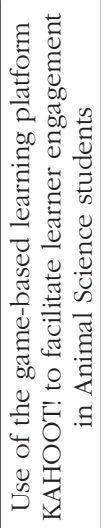 & 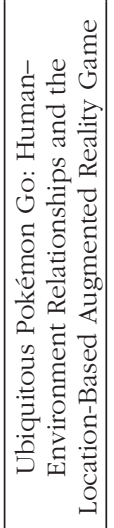 & 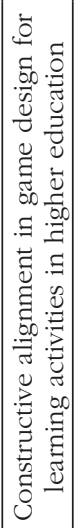 & 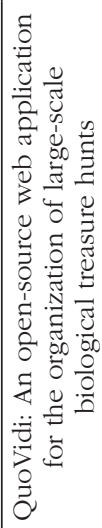 & 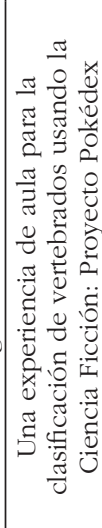 & 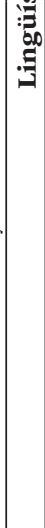 & 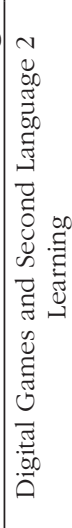 & 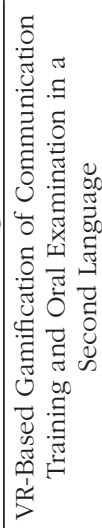 & 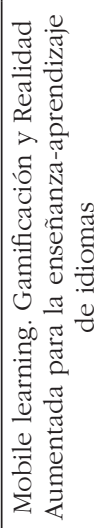 & 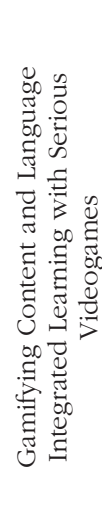 & 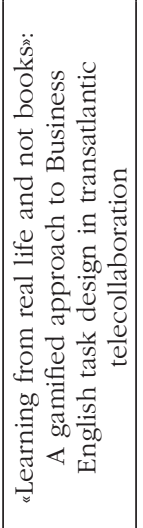 & 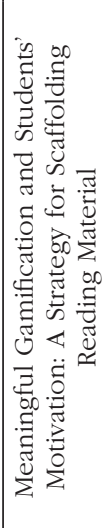 \\
\hline 导 & $\overrightarrow{\vec{c}}$ & ્ָ & ્ֻণ & त్ & त్ & & 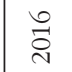 & $\stackrel{0}{\stackrel{0}{0}}$ & $\stackrel{\circ}{\stackrel{0}{c}}$ & $\overrightarrow{\stackrel{\sim}{\sim}}$ & $\overrightarrow{\stackrel{\sim}{\sigma}}$ & $\stackrel{\infty}{\stackrel{\infty}{*}}$ \\
\hline 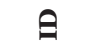 & $y$ & $\sqrt{6}$ & ஜ & $\hat{\sigma}$ & $\infty$ & & హ) & $R$ & $\bar{r}$ & $N$ & & \\
\hline
\end{tabular}


JOEL MANUEL PRIETO ANDREU

REVISIÓN SISTEMÁTICA SOBRE LA EVALUACIÓN DE PROPUESTAS DE GAMIFICACIÓN EN SIETE DISCIPLINAS EDUCATIVAS

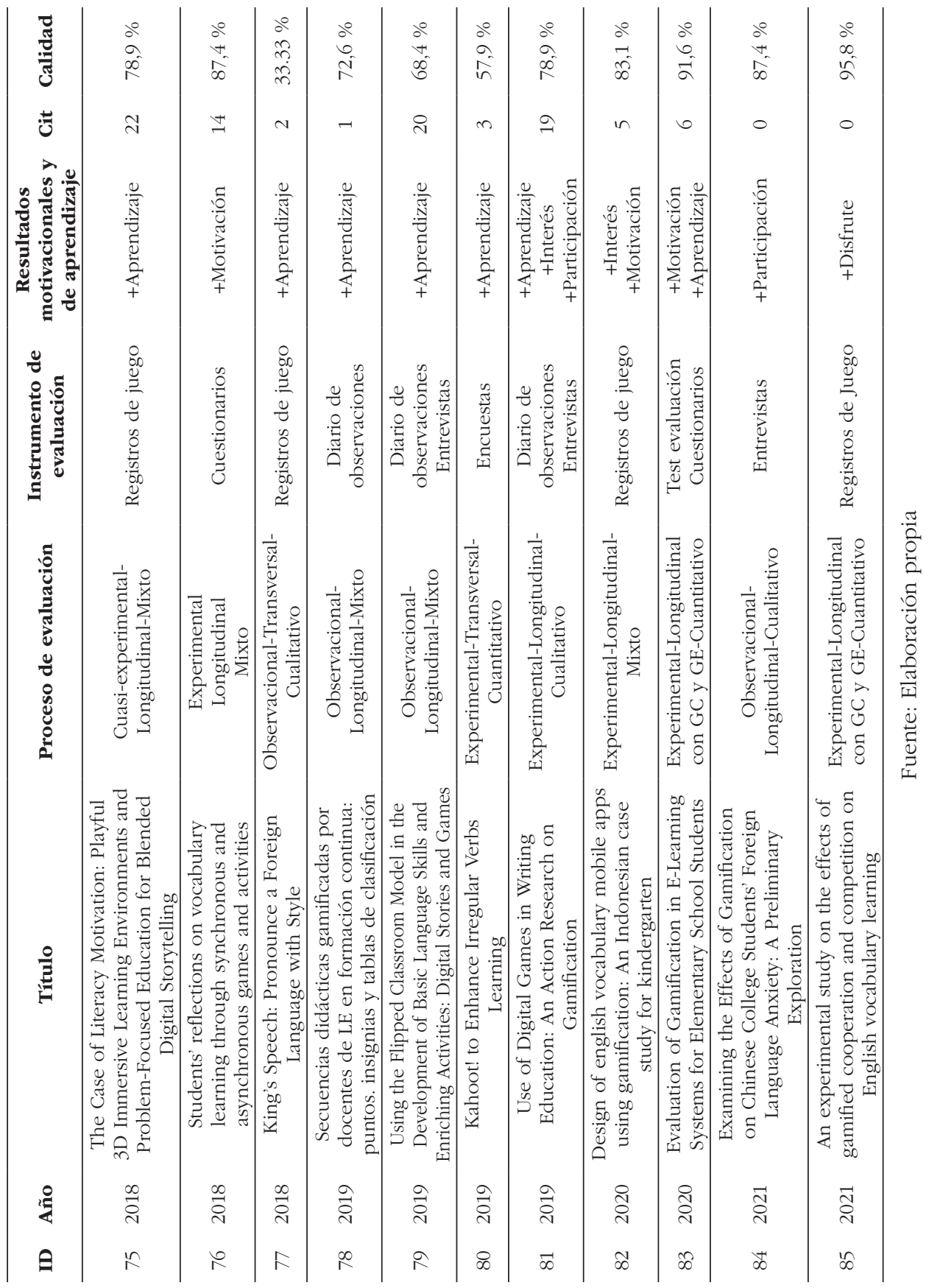

